\title{
Reggie-1 and reggie-2 (flotillins) participate in Rab11a-dependent cargo trafficking, spine synapse formation and LTP-related AMPA receptor (GluA1) surface exposure in mouse hippocampal neurons
}

\author{
Vsevolod Bodrikov ${ }^{\mathrm{a}, *}$, Aline Pauschert ${ }^{\mathrm{a}}$, Gaga Kochlamazashvili ${ }^{\mathrm{b}}$, Claudia A.O. Stuermer ${ }^{\mathrm{a}, *}$ \\ a Dept. Biology, University Konstanz, 78464 Konstanz, Germany

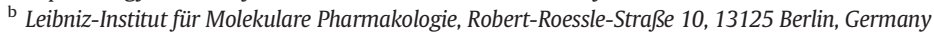

Keywords:

Reggie (flotillin)

Lipid raft

Rab11a

Recycling

Spine synapses

Synaptic plasticity

Knock out mice

Correlative expressional changes

In vitro correlate of LTP

\begin{abstract}
A B S T R A C T
Reggie-1 and -2 (flotillins) reside at recycling vesicles and promote jointly with Rab11a the targeted delivery of cargo. Recycling is essential for synapse formation suggesting that reggies and Rab11a may regulate the development of spine synapses. Recycling vesicles provide cargo for dendritic growth and recycle surface glutamate receptors (AMPAR, GluA) for long-term potentiation (LTP) induced surface exposure. Here, we show reduced number of spine synapses and impairment of an in vitro correlate of LTP in hippocampal neurons from reggie1 k.o. (Flot2-/-) mice maturating in culture. These defects apparently result from reduced trafficking of PSD95 revealed by live imaging of 10 div reggie-1 k.o. (Flot2-/-) neurons and likely impairs co-transport of cargo destined for spines: N-cadherin and the glutamate receptors GluA1 and GluN1. Impaired cargo trafficking and fewer synapses also emerged in reggie-1 siRNA, reggie-2 siRNA, and reggie-1 and -2 siRNA-treated neurons and was in siRNA and k.o. neurons rescued by reggie-1-EGFP and CA-Rab11a-EGFP. While correlative expressional changes of specific synapse proteins were observed in reggie-1 k.o. (Flot2-/-) brains in vivo, this did not occur in neurons maturating in vitro. Our work suggests that reggie-1 and reggie-2 function at Rab11a recycling containers in the transport of PSD-95, N-cadherin, GluA1 and GluN1, and promote (together with significant signaling molecules) spine-directed trafficking, spine synapse formation and the in vitro correlate of LTP.
\end{abstract}

\section{Introduction}

The formation of the axon and elaboration of dendritic arborisation with spines and synapses are regulated by transport of membrane/ membrane proteins to diverse neuronal functional domains. All stages of process development, maturation and adaptive changes of plasticity require membrane and protein recycling and make use of fundamental

Abbreviations: CA, constitutive active; ECS, extracellular solution; EGFP, enhanced green fluorescent protein; EHD, Eps15 homology domain-containing proteins; Flot2, flotillin-2; k.o., knock out; LTP, long term potentiation; MAPK, mitogen-activated kinase; div, days in culture; GluN1, NMDA receptor subunit 1 (NMDA receptor, $N$-Methyl-DAspartate receptor); GluA1, glutamate receptor subunit 1 (AMPA receptor, $\alpha$-amino-3hydroxy-5-methyl-4-isoxazolepropionic acid receptor); RFP, red fluorescent protein; FM4-64FX, fixable analog of FM® 4-64 membrane stain; siRNA, small interfering RNA; pMAPK, phosphorylated MAPK; JNK, Jun kinase; pJUNK, phosphorylated JNK; mAb, monoclonal antibody; pAb, polyclonal antibody; PFA, paraformaldehyde; PBS, phosphate buffered saline; PSD, postsynaptic density; PSD-95, postsynaptic density protein 95; pPSD-95, phosphorylated PSD-95; s.e.m., standard error of the mean; SNX4, sorting nexin4; wt, wild type.

* Corresponding authors.

E-mail addresses: Vsevolod.Bodrikov@uni-konstanz.de (V. Bodrikov), AlinePauschert@web.de (A. Pauschert), kochlamazashvili@fmp-berlin.de (G. Kochlamazashvili), Claudia.Stuermer@uni-konstanz.de (C.A.O. Stuermer). eukaryotic trafficking mechanisms (Park et al., 2004, 2006; Schulte et al., 2010). Indeed, the recycling endosome is involved in the targeted delivery of membrane and proteins to specific sites in the cells (Park et al., 2004, 2006; Solis et al., 2013; Takahashi et al., 2012). In maturated neurons, the recycling endosome contributes to spine growth at glutamatergic synapses and preserves the complex composition of the postsynaptic density (PSD) which harbors cell adhesion proteins, transmitter receptors and molecular scaffolds in register with the presynaptic transmitter-releasing axon protrusions (Kennedy and Ehlers, 2011; Arikkath and Reichardt, 2008). Recycling vesicles not only provide specific compounds for axon and dendritic growth but can serve as reservoir for growth of the PSD and spines, and supply AMPA type glutamate receptors (GluA) for constitutive GluA recycling and long-term potentiation (LTP) induced GluA1 surface exposure (Brown et al., 2007; Park et al., 2004, 2006; Kennedy and Ehlers, 2011). Blocking the transport of the Rab11 recycling endosome not only decreases GluA but also disrupts NMDA receptor (GluN)-dependent delivery of GluA1 to the surface (Park et al., 2004), which impairs LTP.

The lipid raft proteins reggie-1/flotillin-2 and reggie-2/flotillin-1 which reside at synapses (Solis et al., 2010; Suzuki et al., 2011) - were found to bind Rab11a and to participate in recycling and the targeted delivery of membrane/membrane proteins to strategically important 
sites in diverse cells (Stuermer, 2010; Solis et al., 2013). They are essential for growth cone elongation and regeneration in retinal ganglion cells and mouse hippocampal neurons (Munderloh et al., 2009; Koch et al., 2013). They promote the targeted delivery of N-cadherin to the growth cone (Bodrikov et al., 2011) and regulate the targeted recycling of important cell surface proteins to distinct sites of the cell, E-cadherin to adherens junctions (Solis et al., 2013), $\alpha 5$ - and $\beta 1$-integrin to focal adhesions (Hülsbusch et al., 2015), the T cell receptor to the T cell cap (Stuermer et al., 2004, Stuermer, 2010). The targeted delivery seems to be promoted by the interaction of reggie-1 with Rab11a at the recycling compartment (Solis et al., 2013).

That reggies are involved in important steps of synapse formation and plasticity was suggested by reports showing by mass spectrometry analyses that flotillin-1/reggie-2 is upregulated during learning-related events in Aplysia and during spatial memory formation in the mouse hippocampus (Monje et al., 2013). Flotillin-1/reggie-2 is downregulated in the cortical barrel fields after sensory deprivation (Butko et al., 2013). Upregulation of flotillin-1/reggie-2 has, in addition, been implicated in the formation of glutamatergic synapses of mouse hippocampal neurons in vitro (Swanwick et al., 2010) and increase of the frequency of miniature excitatory postsynaptic currents.

Here, we investigated whether reggies participate in Rab11a-dependent trafficking of vesicular carriers in young and maturated mouse hippocampal neurons in culture and whether such trafficking defects would impair spine synapse formation by using neurons from reggie-1 knock out (k.o.; Flot2 -/-) mice (Berger et al., 2013) and neurons after siRNA-mediated reggie downregulation. Indeed, we find that reduced expression of reggie impairs trafficking of PSD-95 (and reggie)decorated vesicular carriers in hippocampal neurons and reduces preand postsynaptic contacts (synapses) in spines. Accordingly, immunostaining intensity for the surface-exposed AMPAR subunit GluA1 was significantly reduced in dendrites particularly after glycine induction of an in vitro correlate of LTP. The reduced spine density in reggie-deficient neurons was rescued by overexpression of constitutive-active (CA) Rab11a consistent with our earlier evidence showing that reggie and Rab11a act in the same pathway for the targeted delivery/recycling (Solis et al., 2013; Stuermer, 2010), which in the present neurons affects PSD-95 and associated cargo destined for the spine synapse.

\section{Material and methods}

\subsection{Animals}

Reggie-1 knock-out (k.o.; Flot2 -/-) C57BL/6] mice were generously provided by Tak W. Mak (The Campbell Family Institute for Breast Cancer Research, University Toronto, Canada; Berger et al., 2013). K.o. and wild type (wt) C57BL/6J mice were raised in the animal facility, University of Konstanz. Male and female mice, 1-2 d and 2 months old, were used.

\subsection{Antibodies}

Monoclonal (mAbs) and polyclonal (pAbs) antibodies were: $\mathrm{mAb}$ against reggie-1 (1:500, ESA-29; Cat\#610383, BD Biosciences Transduction Laboratories), pAb against reggie-1 (1:1000, H-90, Cat\#sc-25507, Santa Cruz), mAb against reggie-2 (1:500, F-3, Cat\#sc-74567, Santa Cruz), mAbs and pAbs against fyn (1:1000, E-3, Cat\#sc-365913 and H80, Cat\#sc-28791, Santa Cruz), active fyn pAb (1:1000, Tyr-527-dephosphorylated, Cat\#2107S, Cell Signaling Technology), pAbs N-cadherin (1:1000, H-63, Cat\#sc-7939, Santa Cruz), pAbs against MAPK/ERK1/2 and pMAPK/pERK1/2 (1:1000, H-72, Cat\#sc-292838 and p-ERK, 1:1000, Thr 202/Tyr 204, Cat\#sc-16982, Santa Cruz), pAb against GluN1 (NMDAR1, 1:1000, D65B7, Cat\# 5704), pAb against SAPK/JNK (Cat\#9252), pAb against T183/Y185-SAPK/JNK (81E11, Cat\# 4668), pAb against Rab11a (1:500, Cat\#2413) and pAb against PSD-95 (1:1000, Cat. N\#2507, Cell Signaling Technology), pAb against GluA1 (AMPAR1, 1:500, Cat\#PC246-100UG, Merck Millipore), pAb against
Rab5a (1:500, Cat\#PAB9011, Abnova), mAb against synaptophysin (1:500, SY38, Cat\#ab8049, Abcam) and pAb against S295-PSD-95 (1:500, EP2615Y, Cat\#ab76108, Abcam). Secondary antibodies rabbit and mouse Ig coupled to HRP, Cy3, or Cy5 were from Dianova. Alexa 488 phalloidin was from Invitrogen.

\subsection{Culture of hippocampal neurons}

Hippocampal neurons from 1 to $2 \mathrm{~d}$ old wt C57BL/6J and reggie- 1 k.o. (Flot2 - /-) mice (Berger et al., 2013) were exposed to papain (Sigma-Aldrich), $30 \mathrm{~min}, 37^{\circ} \mathrm{C}$, centrifuged $\left(80 \times \mathrm{g}, 5 \mathrm{~min}, 37^{\circ} \mathrm{C}\right)$, resuspended and seeded on poly-D-lysine (PDL) coated coverslips (12well plates, Neurobasal A with B-27 serum-free supplement, Life Technologies, and $5 \mu \mathrm{g} / \mathrm{ml} \mathrm{FGF).} \mathrm{After} \mathrm{medium} \mathrm{change,} \mathrm{FGF} \mathrm{was} \mathrm{increased}$ $\left(10 \mu \mathrm{g} / \mathrm{ml}\right.$ ). Cells grew at $37{ }^{\circ} \mathrm{C}$ and $5 \% \mathrm{CO}_{2}$ (Bodrikov et al., 2011).

After fixation, neurons ( $48 \mathrm{~h}$ or $96 \mathrm{~h}$ ) on glass coverslips were stained with Alexa 488 phalloidin (Invitrogen) to measure neurite length (NIH ImageJ software).

\subsection{DNA constructs and transfection}

The reggie- 1 and reggie- 2 siRNAs and the GL2 control siRNA were described previously (Munderloh et al., 2009; Solis et al., 2007). Alexa Fluor 546-labeled siRNA duplexes against reggie-1 (R1.0), reggie-2 (R2.0) and firefly luciferase (GL2, served as nonspecific control) were obtained from Dharmacon. The target sequences were for reggie-1: $5^{\prime}-$ GTTCATGGCAGACACCAAG-3' (R1.0), and for reggie-2: 5'-CACACTGA CCCTCAATGTC-3' (R2.0) and used in wt neurons at concentrations of $9 \mathrm{pmol} / \mathrm{ml}$ (reggie- 1 and reggie-2, respectively, and GL2) or each at $5 \mathrm{pmol} / \mathrm{ml}$ (when reggie-1 and reggie-2 siRNAs were combined, with the GL2 control at $10 \mathrm{pmol} / \mathrm{ml}$ ). Cells were co-transfected with a plasmid encoding PSD-95-EGFP, reggie-1-EGFP, reggie-1-RFP, reggie-2EGFP, CA-Rab11a-EGFP, CA-Rab11a-RFP or EGFP to identify transfected cells (Solis et al., 2013). The reggie-1-EGFP and reggie-2-EGFP plasmids have been used previously for rescue experiments after reggie-1 siRNA and reggie-2 siRNA-mediated downregulation (Solis et al., 2007; Munderloh et al., 2009; Solis et al., 2013). All plasmids were amplified in E. coli and purified by plasmid purification kit. PSD-95-EGFP vector was from Dr. David S. Bredt (Johnson \& Johnson) and kindly provided by Dr. Fukata Masaki (National Institute for Physiological Sciences (NIPS), Aichi, Japan).

Transfections occurred in Optifect Transfection Reagent (Life Technologies).

\subsection{Stimulation of GluN (NMDAR)-mediated exocytosis of GluA1 (AMPAR1)}

LTP was pharmacologically induced in vitro (Ahmad et al., 2012). In brief, medium was removed from coverslips and an extracellular solution (ECS) containing $150 \mathrm{mM} \mathrm{NaCl}, 2 \mathrm{mM} \mathrm{CaCl} 2,5 \mathrm{mM} \mathrm{KCl}, 10 \mathrm{mM}$ HEPES, $30 \mathrm{mM}$ glucose, $0.001 \mathrm{mM}$ tetrodotoxin (TTX), $0.01 \mathrm{mM}$ strychnine and $0.03 \mathrm{mM}$ bicuculline was added (RT). To stimulate NMDA receptors (GluN), samples were incubated for $3 \mathrm{~min}$ at RT with ECS containing $300 \mu \mathrm{M}$ glycine. ECS without glycine served as control. After washes, ECS without glycine was added for $30 \mathrm{~min}$ at RT. Neurons were fixed in 4\% PFA (PBS, pH 7.3, 15 min, on ice; Jurado et al., 2013), washed with PBS, and blocked in 3\% BSA. GluA1 pAb was applied in PBS and 3\% BSA to fixed and non-permeabilized cells.

\subsection{FM4-64FX uptake}

Wt hippocampal neurons, maturated for $8 \mathrm{~d}$ in vitro (div) were cotransfected with reggie- 1 siRNA or control siRNA together with PSD95-EGFP, CA-Rab11a-EGFP or EGFP. After $2 \mathrm{~d}$ cells were loaded with $10 \mu \mathrm{M}$ FM4-64FX dye (Invitrogen) in depolarizing buffer $(100 \mathrm{mM}$ $\mathrm{NaCl}, 50 \mathrm{~mm} \mathrm{KCl}, 2 \mathrm{mM} \mathrm{CaCl}, 1 \mathrm{mM} \mathrm{MgCl}, 10 \mathrm{mM}$ glucose, $15 \mathrm{mM}$ 
HEPES, pH 7.3) for $1 \mathrm{~min}$ to load vesicles. Then coverslips were transferred to wash buffer $\left(140 \mathrm{mM} \mathrm{NaCl}, 5 \mathrm{mM} \mathrm{KCl}, 2 \mathrm{mM} \mathrm{CaCl}_{2}, 1 \mathrm{mM}\right.$ $\mathrm{MgCl}_{2}, 10 \mathrm{mM}$ glucose, $15 \mathrm{mM}$ HEPES, $\mathrm{pH}$ 7.3) for $5 \mathrm{~min}$ to remove excess dye and fixed in $4 \%$ PFA for 10 min. After fixation coverslips were washed with PBS and mounted with Mowiol. FM4-64FX dye uptake was analysed by using Scion image software (Scion Corporation).

\subsection{Immunolabeling}

Neurons were fixed in 4\% PFA (PBS, pH 7.3, 15 min at room temperature (RT)), washed, and blocked in 3\% BSA. All Abs (except for pAb against GluA1, see above) were applied to fixed cells after permeabilization in $0.25 \%$ Triton X-100 (PBS, 5 min, RT). Abs applied in 3\% BSA in PBS $\left(2 \mathrm{~h}, 37^{\circ} \mathrm{C}\right.$ ) were detected with secondary Abs (45 min, RT; Bodrikov et al., 2011).

\subsection{Analysis of PSD-95 trafficking in live neurons}

Neurons were co-transfected with reggie-1 siRNA or reggie-2 siRNA or reggie-1 and -2 siRNAs combined or with control siRNA, together with EGFP, PSD-95-EGFP, reggie-1-RFP, or CA-Rab11a-EGFP, $8 \mathrm{~d}$ after plating. Recordings of live neurons were performed using a Zeiss Cellobserver HS with a Spinning Disk imaging system equipped with an alpha-Plan-Fluor $100 \times / 1.45$ Objective (Zeiss). During recordings, neurons were kept at $37{ }^{\circ} \mathrm{C}$ and $5 \% \mathrm{CO}_{2}$. Images were acquired at $1 \mathrm{~s}$ intervals over $3 \mathrm{~min}$. The same methods were used to record vesicle trafficking in 4 div wt and reggie-1 k.o. (Flot2-/-) neurons.

\subsection{Analysis of number of spines of hippocampal neurons in vitro}

Two d after transfection, neurons were fixed in 4\% PFA (10 min). All Z-stacks were taken at the confocal microscope LSM700 META (Carl Zeiss Germany) using the same settings and the Zeiss Zen program. Images were analysed with ImageJ. The length of neurites was measured by the program and the number of spines was counted. A spine was defined as a protrusion from a dendrite, shorter than $5 \mu \mathrm{m}$ and devoid of branching. Number of spines was set in relation to neurite length to estimate spine formation.

\subsection{Visualization and image processing}

Immunostained samples were visualized at the LSM with a $63 \times / 1.4$ oil immersion lens. Images were further processed using Scion Image (Scion Corporation) or NIH ImageJ software. The analysis of protein colocalisation was performed as described previously (Bodrikov et al., 2011). In brief, clusters of interest (reggie-1, reggie-2, PSD-95 or Rab11a) were defined by increased immunofluorescence of at least $30 \%$ above background. These clusters were automatically outlined using the threshold function of the Scion Image software (Scion Corporation). Within the outlined areas the mean intensities of fluorescence obtained by Abs against GluN1, GluA1, PSD-95, N-cadherin, Rab-11a or Rab-5a and associated with clusters of interest were measured. The same threshold was used for all groups. All experiments were performed two to three times. Co-localisation profiles were plotted using $\mathrm{NIH}$ ImageJ software.

\subsection{Immunofluorescence quantification}

Distribution and mean immunofluorescence intensities along neurites were determined by ImageJ. The mean intensity of outlined neurites was measured and the average mean intensity between the control and experimental groups was compared. The correlation coefficients between distributions of reggie-1 and -2, PSD-95, GluA1, GluN1 were analysed.

\subsection{Gel electrophoresis and immunoblotting}

Brain homogenates (50 mM Tris- $\mathrm{HCl}$ buffer, $\mathrm{pH} 7.5,1 \mathrm{mM} \mathrm{CaCl}_{2}$, $1 \mathrm{mM} \mathrm{MgCl}_{2}$, and $1 \mathrm{mM} \mathrm{NaHCO}_{3}$ ) were lysed ( $40 \mathrm{~min}$ ) on ice (RIPA buffer, Roche Diagnostics) for gel electrophoresis. Proteins, separated by $8 \%$ or $10 \%$ SDS-PAGE were electroblotted onto nitrocellulose transfer membrane (PROTRAN; Schleicher \& Schuell) overnight at $5 \mathrm{~mA}$. Immunoblots were incubated in primary Abs and peroxidase-conjugated secondary Abs and visualized with SuperSignal (West Pico reagents; Pierce) on BIOMAX film (Sigma-Aldrich) together with MW markers (Bio-Rad Laboratories). Chemiluminescence was quantified using TINA 2.09 software (University of Manchester, UK). Intensities were normalized to $\alpha$-tubulin loading controls.

Cell cultures of 5 div hippocampal neurons transfected with reggie-1 and -2 siRNAs (combined) were lysed ( $30 \mathrm{~min}$ ) on ice in EDTA-free RIPA buffer. Proteins were separated by $10 \%$ SDS-PAGE, electroblotted and incubated in primary Abs and peroxidase-conjugated secondary Abs (see above).

\subsection{Data analysis}

Values are expressed as mean values \pm SEM (SIGMA Plot, from at least three independent experiments). Statistical analysis was performed by two-tailed unpaired Student's t-test or One-way ANOVA with Multiple Comparisons (and pairwise comparisons). Values of $\mathrm{p}<0.05$ were statistically significant (EXCEL, SIGMA Plot).

\section{Results}

3.1. Reggie-1 and reggie-2 together with Rab11a regulate the number of spine synapses in hippocampal neurons in vitro

To determine whether reggies contribute to spine formation (Fig. 1), we transfected 8 div neurons with EGFP (to visualize their morphology), and reggie-1 (or else reggie-2) siRNA and control siRNA (Munderloh et al., 2009), respectively. That the siRNAs are indeed specific for reggie-1 and reggie-2, respectively, has been shown previously (Bodrikov et al., 2011; Munderloh et al., 2009; Solis et al., 2013) and was confirmed in the context of Western blot experiments after application of reggie-1 and -2 siRNAs combined in hippocampal neurons (Fig. $1 \mathrm{H}$ ) and by rescue experiments in which siRNA-treated neurons were co-transfected with siRNA-resistant reggie-1-EGFP and reggie-2-EGFP (Fig. $1 \mathrm{~A}-\mathrm{F}$ ), respectively (Munderloh et al., 2009; Solis et al., 2013). Interestingly, in contrast to Hela cells (Solis et al., 2007), reggie-1 siRNA did not lead to a reduction in reggie-2 protein (Fig. $1 \mathrm{I}, \mathrm{J}$ ).

SiRNA transfected neurons showed a significant reduction in number of spines of $24 \%$ (reggie-1 siRNA) and 58\% (reggie-2 siRNA) compared to control siRNA-treated neurons (Fig. 1 A, B, C, D). This reduction in spine number was rescued by co-transfecting neurons with reggie-1 siRNA and reggie-1-EGFP which resulted in an increase in spine number of $103 \%$ (Fig. $1 \mathrm{~A}, \mathrm{~B}$ ). With reggie-2 siRNA and reggie2-EGFP spine number raised to $106 \%$ (Fig. 1 C, D). Thus, reggie-1 and reggie-2 significantly affect number of spines when acutely downregulated by siRNAs. Yet, in 10 div hippocampal neurons from reggie-1 k.o. (Flot2-/-) mice (Fig. 1 E, F) number of spines was not reduced. However, the number of spine synapses fell significantly (see below). In these reggie-1 k.o. (Flot2-/-) neurons, reggie-1 siRNA had no effect on spine number (Fig. $1 \mathrm{E}$ ) so that the siRNA against reggie-1 does not seem to have unspecific side effects.

Rab11a is known to interact with reggies in Hela and A431 cells and to rescue cargo trafficking defects (Hülsbusch et al., 2015; Solis et al., 2013). Here we show that Rab11a is co-localised with reggie-1 and reggie-2 in hippocampal neurons in dendrites and spines (Fig. $1 \mathrm{G}$ ). To determine whether CA-Rab11a would rescue the decrease in spine number after reggie- 1 and reggie-2 siRNA transfection in 10 div neurons, we quantified number of spines in neurons co-transfected with 
either control siRNA, reggie-1 siRNA or reggie-2 siRNA and CA-Rab11aEGFP. Neurons transfected with control siRNA and CA-Rab11-EGFP compared to control siRNA and EGFP-transfected counterparts showed a $45.5 \%$ increase in spine number (Fig. 1 B). As demonstrated in Fig. 1 (A-D) the reduction in number of spines by knock down of reggie-1 or reggie-2 was rescued by co-transfection with CA-Rab11a. Thus, reggie-1, reggie-2 and Rab11a function in the same trafficking pathway for the transport of vesicular carriers in dendrites and spines.

\subsubsection{PSD-95 and PSD-destined cargo in reggie-1 and -2/Rab11a vesicular carriers in dendrites and spines}

Next, we examined by immunostaining with specific antibodies whether reggie-1 and Rab11a-labeled vesicles carry cargo destined for the PSD in spines. In 5 div neurons $(\mathrm{n}=75)$, reggie- 1 and -2 were colocalised with Rab11a (52\% and 49\%, respectively), PSD-95 (35\% and $58 \%$, respectively), N-cadherin (31\% and $27 \%$, respectively), GluA1 (38\% and $47 \%$, respectively) and GluN1 (40\% and 45\%, respectively, Fig. 1 G). Consistent with earlier results (Solis et al., 2013), reggie-1 and -2 were not co-localised to any significant extent with Rab5a (11.5\% and 7.7\%, respectively) which served as control.

To examine whether a reduction in reggie protein expression level by reggie- 1 siRNA and reggie-2 siRNA would affect the stability of the proteins transported in reggie vesicles, Western blots of cultured neurons treated with reggie- 1 and reggie-2 siRNAs combined were performed. While reggie- 1 and reggie- 2 siRNAs reduced the reggie- 1 and reggie-2 protein levels significantly (Fig. $1 \mathrm{H}$ ), the expression of PSD95, N-cadherin, GluA1 and GluN1 was unchanged compared to control siRNA-transfected neurons. Reggie-1 siRNA, however, did not affect reggie-2 expression as determined by quantifying immunostaining intensities (Fig. $1 \mathrm{I}, \mathrm{J}$ ) - in contrast to the situation in Hela cells (Solis et al., 2007).

To determine if PSD-95 is transported in reggie vesicles, we double transfected 10 div neurons with reggie-1-RFP and PSD-95-EGFP and monitored vesicle trafficking. $76.7 \%$ of reggie- 1 positive trafficking vesicles contained PSD-95 (Fig. 2 A, B) suggesting that reggie-1 and PSD-95 traffic together and that reggie-1 might influence the PSD-95 mediated increase in spine synapses.

\subsubsection{Reggie-1 and Rab11a affect number of synapses}

To determine if reggie-1 would affect synapse number we cotransfected neurons with PSD-95-EGFP, or EGFP as control, and reggie-1 siRNA, or control siRNA, and exposed these neurons to FM464FX (which is endocytosed presynaptically and serves in our present experiments simply as a marker for the axonal endings on spines). In neurons transfected with reggie-1 siRNA, the FM4-64FX immunofluorescence intensity was reduced by $47 \%$ (Fig. 2 C, D). It was rescued by co-transfecting neurons with reggie-1 siRNA and reggie-1-EGFP (Fig. 2 C, D). In neurons transfected with PSD-95-EGFP, known to promote spine formation (Charych et al., 2006), the FM4-64FX labeling increased significantly $(+92 \%)$ over EGFP-transfected neurons (Fig. 2 C, D). In PSD-95-EGFP and reggie-1 siRNA double transfected neurons, however, the FM4-64FX fluorescence intensity was reduced by 33\% (Fig. 2 C, E) indicating that siRNA-mediated reduction of reggie-1 reduces the PSD95-EGFP-induced increase in spine synapses. The finding that PSD-95 overexpression increases the FM4-64FX intensity and thus labeled synapses - which were reduced by the loss of reggie-1 - suggests that reggie-1 indeed affects the postsynaptic spine (see Discussion). These results are consistent with a function of reggie-1 in PSD-95 transport to synapses.

To determine if the reduction in synapses (FM4-64FX intensity) by reggie-1 siRNA can be cross-rescued by Rab11a, neurons were cotransfected with reggie-1 siRNA and CA-Rab11a-EGFP. Indeed, CARab11a rescued the reggie-1 siRNA-induced reduction of FM4-64FX uptake by $106 \%$ over EGFP and control siRNA-transfected neurons (Fig. 2 C, F). Thus, Rab11a and reggie-1 are involved in the generation of spine synapses in hippocampal neurons by regulating trafficking of the recycling endosome and the delivery of PSD-95 as one important cargo molecule for spines.

\subsection{Defects in PSD-95 trafficking and reduced number of synapses in hippo-} campal neurons from reggie-1 k.o. (Flot2-/-) mice.

To understand in greater detail how reggies might regulate spine synapse formation, we isolated hippocampal neurons from reggie-1 k.o. (Flot2-/-) mice (Berger et al., 2013) and analysed trafficking

Fig. 1. Reggie-1 and reggie-2 regulate spines together with Rab11a and co-localise at Rab11a-labeled vesicular carriers transporting cargo destined for the PSD in spines (A) Hippocampal neurons at 10 div were transfected with reggie-1 siRNA (R1siRNA) or control siRNA (contr siRNA) and double transfected with EGFP (to visualize morphology, upper row), or with reggie1(R1)-EGFP as rescue, or with CA-Rab11a-EGFP. Portions of dendrites are shown at higher magnification in the second row. Number of spines over dendrites was quantified. Scale bar, 20 um in upper; 5 um in lower row of images. (B) Spine number was 26\% reduced (statistically significant in reggie-1 siRNA compared to control siRNA-transfected neurons: N, spines: $31.9 \pm 1.21$ [mean \pm s.e.m.] in control siRNA/EGFP and $23.7 \pm 1.23$ [mean \pm s.e.m.] in reggie-1 (R1) siRNA/EGFP-treated neurons, $\mathrm{n}=406$ and $\left.\mathrm{n}=322,{ }^{* * *} \mathrm{p}<0.001\right)$. Number of spines was rescued by R1-EGFP (N, spines: $31.9 \pm 1.21$ [mean \pm s.e.m.] in control siRNA and $33.0 \pm 1.97$ [mean \pm s.e.m.] in R1 siRNA-treated neurons, $\mathrm{n}=406$ and $\mathrm{n}=303, \mathrm{p}=$ 0.69 n.s.) and by CA-Rab11a-EGFP (N, spines: $31.9 \pm 1.21$ [mean \pm s.e.m.] in control siRNA and $29.6 \pm 3.0$ [mean \pm s.e.m.] in R1 siRNA-treated neurons, $\mathrm{n}=406$ and $\mathrm{n}=437, \mathrm{p}=$ 0.72 n.s.). Spine number was $45.5 \%$ increased by CA-Rab11a-EGFP in control siRNA-transfected neurons ( $\mathrm{N}$, spines: $31.9 \pm 1.21$ [mean \pm s.e.m.] in control siRNA/EGFP and $46.3 \pm 4.6$ [mean \pm s.e.m.] in control siRNA/CA-Rab11a-EGFP-treated neurons, $\mathrm{n}=406$ and $\mathrm{n}=350,{ }^{* * *} \mathrm{p}<0.001$ ). n.s., not significant. One-way ANOVA. Multiple Comparisons versus Control Group (Holm-Sidak method). (C) Reggie-2 (R2) siRNA causes a loss of spines which is rescued by R2-EGFP and CA-Rab11a-EGFP. Scale bar, 10 um. (D) Quantification of spines and the statistically significant $58 \%$ reduction after reggie-2 (R2) siRNA (N, spines: $13.7 \pm 0.57$ [mean \pm s.e.m.] in control siRNA and $5.7 \pm 0.36$ [mean \pm s.e.m.] in R2 siRNA-treated neurons, $\mathrm{n}=383$ and $\left.\mathrm{n}=352,{ }^{* * *} \mathrm{p}<0.001\right)$. The reduction in spine number was rescued by R2-EGFP ( $\mathrm{N}$, spines: $13.7 \pm 0.57$ [mean \pm s.e.m.] in control siRNA and $14.5 \pm 0.67$ [mean \pm s.e.m.] in R2 siRNA-treated neurons, $\mathrm{n}=383$ and $\mathrm{n}=309, \mathrm{p}=0.32 \mathrm{n} . \mathrm{s}$.) and by CA-Rab11a-EGFP ( $\mathrm{N}$, spines: $13.7 \pm 0.57$ [mean \pm s.e.m.] in control siRNA and $11.3 \pm 1.36$ [mean \pm s.e.m.] in R2 siRNA-treated neurons, $\mathrm{n}=383$ and $\mathrm{n}=281, \mathrm{p}=0.12 \mathrm{n}$.s.). One-way ANOVA. Multiple Comparisons versus Control Group (Holm-Sidak method). (E) Neurons from reggie-1 (R1) k.o. (Flot2 -/-) mice at 10 div (transfected with EGFP to visualize cell morphology), did not exhibit a difference in number of spines (F) when compared to wt hippocampal neurons. No change in number of spines was observed when R1 k.o. (Flot2-/-) neurons were transfected with R1 siRNA. Scale bar, $50 \mu \mathrm{m}$ in upper and $10 \mu \mathrm{m}$ in lower row of images. (F) Quantification of spine number ( $\mathrm{N}$, spines: $10.4 \pm 0.46$ [mean \pm s.e.m.] in wt and $9.52 \pm 0.54$ [mean \pm s.e.m.] in R1 k.o. (Flot2 $-/-$ ) neurons, $\mathrm{n}=453$ and $\mathrm{n}=$ 437, $\mathrm{p}=0.22$ n.s.). Spine number in R1 k.o. (Flot2 -/-) neurons was not affected by R1 siRNA (N, spines: $10.4 \pm 0.46$ [mean \pm s.e.m.] in R1 k.o. (Flot2 $-/-$ ) and $9.86 \pm 0.68$ $[$ mean \pm s.e.m.] in R1 k.o./R1 siRNA neurons, $\mathrm{n}=453$ and $\mathrm{n}=249, \mathrm{p}=0.51$ n.s.). One-way ANOVA. Multiple Comparisons versus Control Group (Holm-Sidak method). (G) Double immunostainings were performed in hippocampal neurons pairwise with specific Abs against reggie-1, reggie-2 (green, middle column), N-cadherin (N-Cad) or PSD-95 (green, middle column) in combination with specific Abs against (red, left column): GluA1, GluN1, PSD-95, Rab11a and Rab5a as control. Third column shows the merge and vesicular carriers (white arrows) with two co-localised cargos (yellow). Number of neurons, $\mathrm{n}=75$ in 3 independent experiments. Pairs and co-localisations support the existence of reggie-1/Rab11acoated carriers with cargo significant for the PSD. Scale bar, $5 \mu \mathrm{m}$. (H) Signals on Western blots for the indicated proteins were quantified over three independent experiments and depicted in the histograms to the right with indications of statistically significant differences between the pairs (2-tailed unpaired Student's $t$-test). Wt hippocampal neurons cultured for $5 \mathrm{~d}$ and treated with control (contr) or reggie-1 and reggie-2 (R1/R2) siRNAs (combined) showed no change in PSD-95 (25.8 \pm 5.5 [mean \pm s.e.m.] in control siRNA and $24.4 \pm$ 5.3 [mean \pm s.e.m.] in R1/2 siRNA-treated neurons, $p=0.86$, n.s.), in GluN1 (28.4 \pm 5.4 [mean \pm s.e.m.] in control siRNA and $26.4 \pm 5.0$ [mean \pm s.e.m.] in R1/2 siRNA-treated neurons, $\mathrm{p}=0.79$, n.s.), in GluN1 (25.6 \pm 3.2 [mean \pm s.e.m.] in control siRNA and $24.6 \pm 3.8$ [mean \pm s.e.m.] in R1/2 siRNA-treated neurons, $\mathrm{p}=0.79$, n.s.), in N-cadherin ( $31.9 \pm$ 7.4 [mean \pm s.e.m.] in control siRNA and $33.4 \pm 6.8$ [mean \pm s.e.m.] in R1/2 siRNA-treated neurons, $\mathrm{p}=0.79$, n.s.). Reggie-1 was significantly downregulated by R1/2 siRNAs ( $24.5 \pm$ 5.3 [mean \pm s.e.m.] in control siRNA and $5.6 \pm 2.3$ [mean \pm s.e.m.] in R1/2 siRNA-treated neurons, ${ }^{*} \mathrm{p}<0.05$ ), and reggie-2 was significantly downregulated by R1/2 siRNAs ( $32.3 \pm$ 4.5 [mean \pm s.e.m.] in control siRNA and $3.5 \pm 1.9$ [mean \pm s.e.m.] in R1/2 siRNA-treated neurons, ${ }^{*} \mathrm{p}<0.05$ ). $\alpha$-tubulin served as loading control. (I) Immunostainings with specific Abs against reggie- 2 were performed in hippocampal neurons treated with control or reggie- 1 siRNA. Number of neurons, $n=150$ in 3 independent experiments. (J) Quantification of reggie-2 immunostainings showed no decrease of reggie-2 after R1 downregulation ( $37.8 \pm 3.0$ [mean \pm s.e.m.] in control siRNA and $38.9 \pm 4.1$ [mean \pm s.e.m.] in R1 siRNA-treated neurons, $\mathrm{p}=0.84, \mathrm{n} . \mathrm{s}$.) (2-tailed unpaired Student's $t$-test). 
A

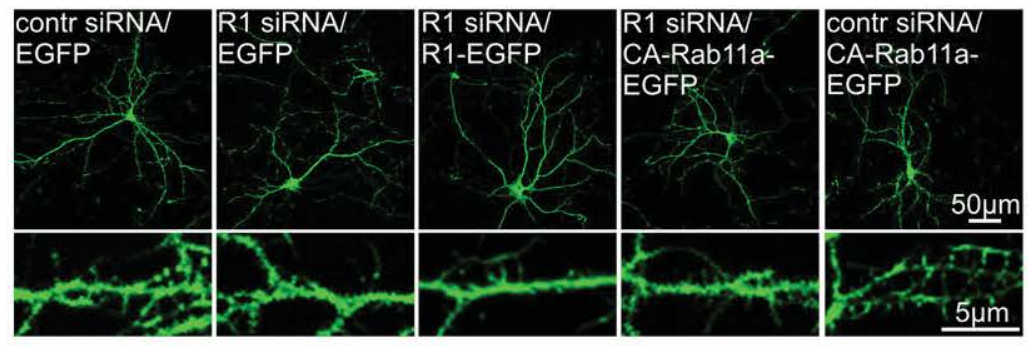

B
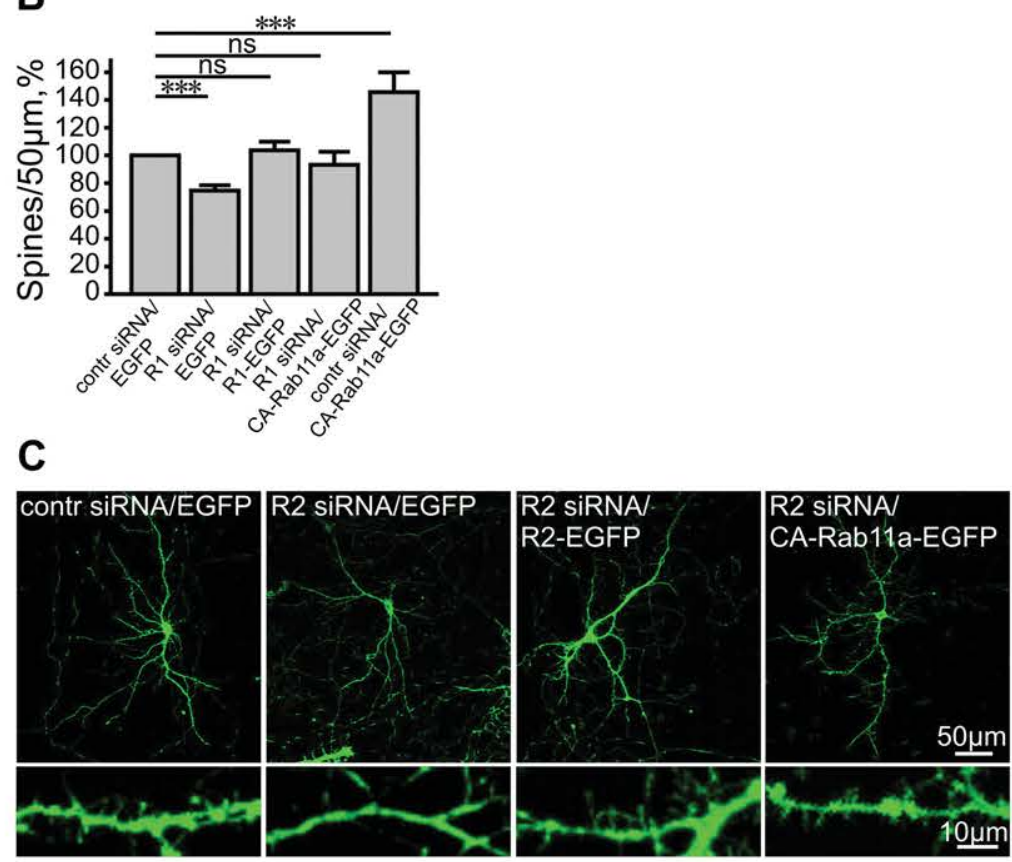

D

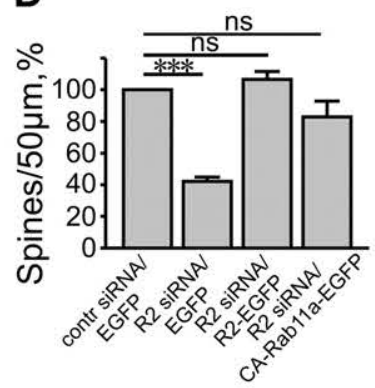

E

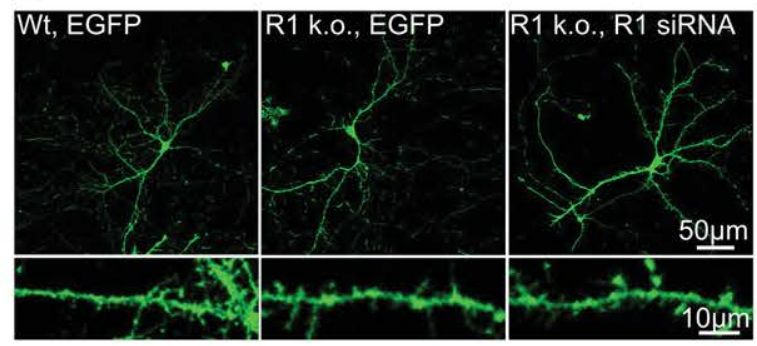

F

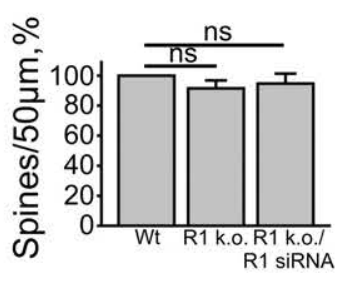


G

\begin{tabular}{|c|c|c|}
\hline GluA1 _... & Reggie-1 & $\operatorname{merg}_{\infty}+ \pm \neq \ldots \ldots$ \\
\hline luN1 & leggie-1,+ & merge \\
\hline SD-95 & Reggie-1,$\cdots$ & merge \\
\hline ab11a & Reggie-1 & merge $t^{t}-\pi, \ldots+t, t$. \\
\hline IuA1 & Reggie-2 , , & merge $i t \ldots+\ldots$ \\
\hline olun1 20 & 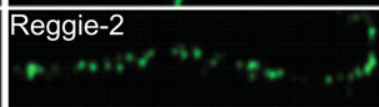 & 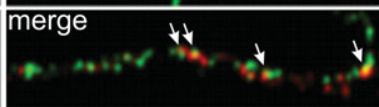 \\
\hline 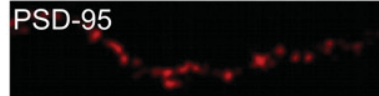 & Reggie-2 & nerge \\
\hline Rab11a & Reggie-2 & merge \\
\hline$\cdots$ & N-Cad $+x$ & $\operatorname{merg}_{i} \pm t, t$ \\
\hline Rab11a & $\mathrm{N}-\mathrm{Cad}, \ldots$ & 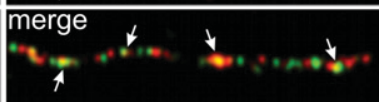 \\
\hline Rab11a & $\begin{array}{c}\text { PSD-95 } \\
-\ldots+\ldots 6\end{array}$ & merge $t \downarrow \cdots+. t$ \\
\hline Rab5a,$\ldots \ldots$ & Reggie-1 , $\cdots \cdots \infty$ & $\int_{0}^{\text {merge } \ldots \ldots \ldots . . .}$ \\
\hline Rab5a & Reggie-2 $\ldots$ & merge \\
\hline
\end{tabular}

H

control R1/R2

siRNA siRNA

PSD-95 $=0$

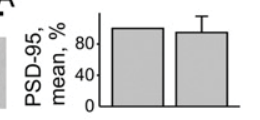

control R1/R2

SiRNA SiRNA

GluN1 $\ldots \ldots$

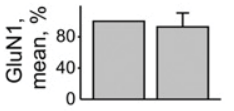

Reggie-1

$-1$

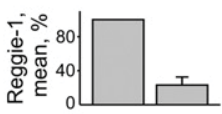

Reggie-2

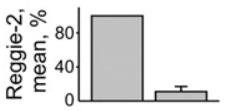

GluA1

$\alpha$-tubulin

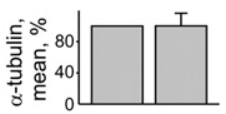

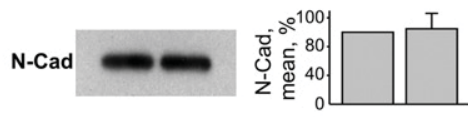

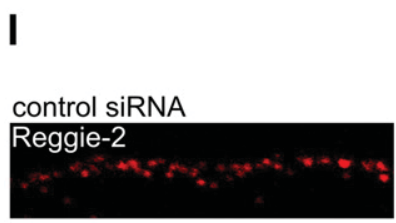

R1 siRNA

Reggie-2
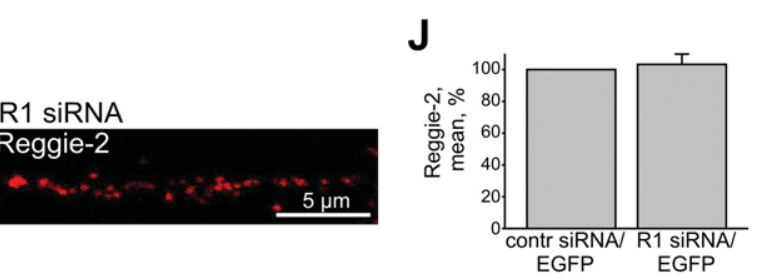

Fig. 1 (continued). 


\section{A}

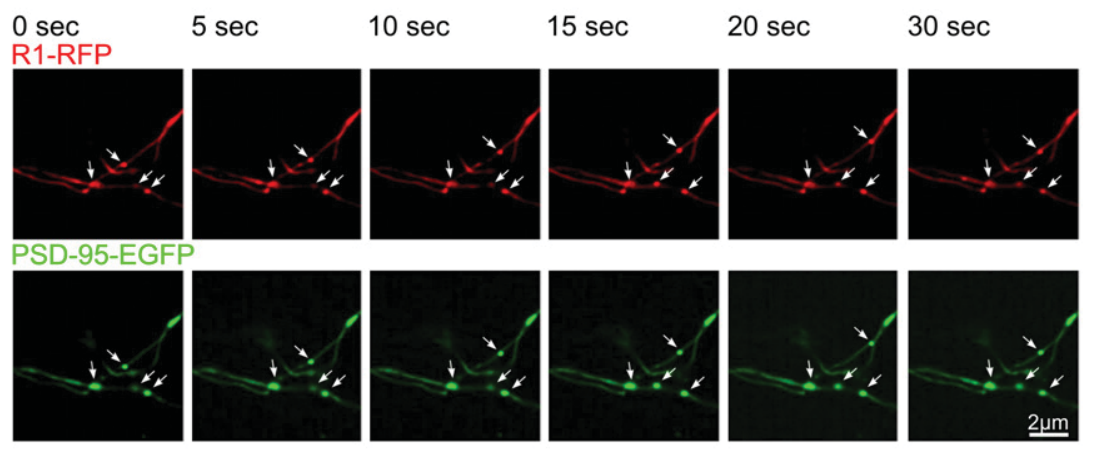

B

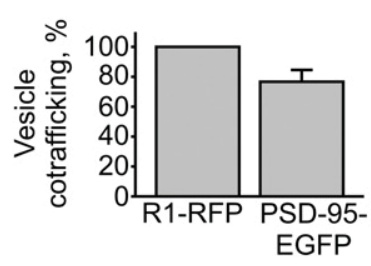

C

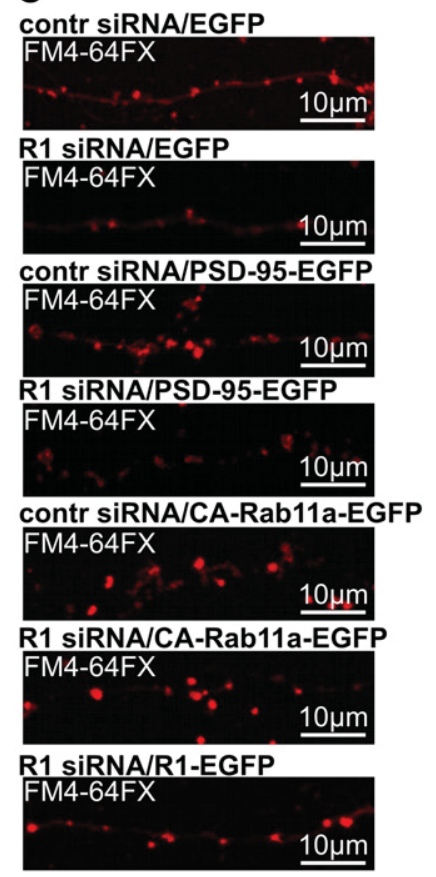

D

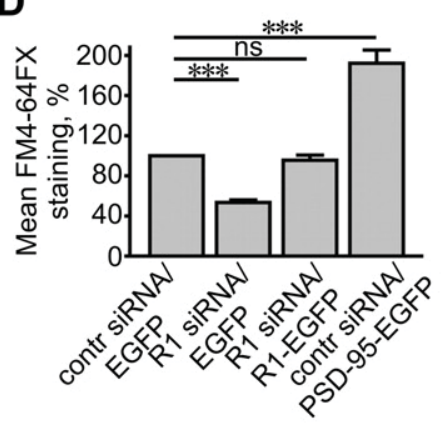

E

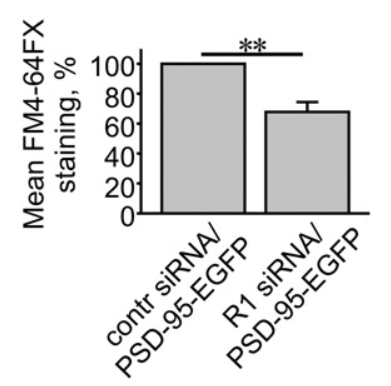

F

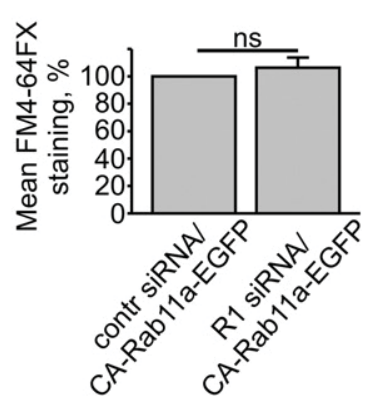

Fig. 2. Reggie-1 is involved in PSD-95 trafficking and regulates together with Rab11a spine synapses. (A) Images from a continuous movie of 3 min showing the movement of vesicles over 30 s co-transporting reggie-1 (R1-RFP, arrows) and PSD-95-EGFP (arrows). Scale bar, 2 um. (B) Moving R1-RFP positive vesicles were analysed and vesicles containing R1-RFP and PSD-95EGFP quantified. 76.7\% of the R1-RFP vesicles had PSD-95-EGFP. N, vesicles = 93; number of neurons, $n=32$ in 2 independent experiments. (C) Putative spine synapses were visualized by FM4-64FX fluorescence in neurons treated with reggie-1 (R1, or control) siRNA and expressing either EGFP, PSD-95-EGFP, CA-Rab11a-EGFP or R1-EGFP (rescue). Scale bar, 10 $\mu$ m. (D) Spine synapse number was 47\% reduced in R1siRNA-treated neurons (mean fluorescence intensity of FM4-64FX: $35.4 \pm 1.25$ [mean \pm s.e.m.] in control siRNA/EGFP and $1.89 \pm 0.9$ [mean \pm s.e.m.] in R1 siRNA/EGFP-treated neurons, ${ }^{* * *}$ p $<0.001$ ). Spines, $\mathrm{N}=527$ and $\mathrm{N}=490$, in 3 independent experiments. Number of spines was rescued by R1-EGFP ( $\mathrm{N}$, spines: $35.4 \pm 1.25$ [mean \pm s.e.m.] in control siRNA/EGFP and $33.8 \pm 1.79$ [mean \pm s.e.m.] in R1siRNA/R1-EGFP-treated neurons, $n=490$ and $n=252, p=0.46$ n.s.) and significantly increased after PSD-95-EGFP overexpression (92\%, N, spines: $35.4 \pm 1.25$ [mean \pm s.e.m.] in control siRNA and $68.0 \pm 4.6$ [mean \pm s.e.m.] in control siRNA/PSD-95-EGFP-treated neurons, $\mathrm{n}=490$ and $\mathrm{n}=306,{ }^{*} * \mathrm{p}<<0.001$ ). One-way ANOVA. Multiple Comparisons versus Control Group (Holm-Sidak method). (E) PSD-95-EGFP did not rescue the spine synapse loss $\left(15.9 \pm 1.6\right.$ [mean \pm s.e.m.] in control siRNA and $10.8 \pm 1.05$ [mean \pm s.e.m.] in R1siRNA-treated neurons, $\left.{ }^{* *} \mathrm{p}<0.01\right)$. Neurons, $\mathrm{n}=397$ and $\mathrm{n}=375$, in 3 independent experiments. (F) Cotransfection of the neurons with CA-Rab11a-EGFP rescued the reduction in spine synapses (mean fluorescence intensity of FM4-64FX: $1.23 \pm 0.085$ [mean \pm s.e.m.] in cotransfected control siRNA and $1.31 \pm 0.091$ [mean \pm s.e.m.] in cotransfected R1 siRNA-treated neurons, $\mathrm{p}=0.54$, n.s.). Number of neurons, $\mathrm{n}=422$ and $\mathrm{n}=416$ in 3 independent experiments (2-tailed unpaired Student's $t$-test).

and emergence of LTP-induced AMPAR GluA1 on dendrites and spines. As reggie-1 k.o. (Flot2-/-) mice have no overt phenotype (Berger et al., 2013; Bitsikas et al., 2014a,b) we also investigated whether the brain might have strategies to compensate the loss of reggie-1.
When neurons of 1-2 d postnatal reggie-1 k.o. (Flot2-/-) mice were plated and compared to age-matched wt neurons, process outgrowth was significantly delayed over the first $24 \mathrm{~h}$ in culture by $21 \%$ but at 4 div, reggie- 1 k.o. (Flot $2-/-$ ) neurons were differentiated to 
the same extent as wt neurons (Fig. $3 \mathrm{~A}, \mathrm{~B}, \mathrm{C}$ ) indicating that they compensated the initial delay in process extension.

To determine whether reggie-1 k.o. (Flot2-/-) neurons would possess fewer spine synapses, we performed immunostainings with synaptophysin and PSD-95 Abs, and synaptophysin and GluN1 Abs, respectively (for pre and postsynaptic elements, Fig. 3 D, G). Quantification of staining intensity showed that PSD-95 was 53\% and GluN1 55\% reduced in neurons from reggie- 1 k.o. (Flot2-/-) mice compared to wt controls (Fig. 3 E, F, H, I). Moreover, co-localisation of synaptophysin and PSD-95 (indicative of pre- and postsynaptic elements of spines) was reduced by $56 \%$ in reggie- 1 k.o. (Flot $2-/-$ ) neurons over wt controls, and synaptophysin and GluN1 by $42 \%$ (Fig. 3 F, I). Thus, reggie-1 k.o. leads to an abnormal distribution of PSD-95 in dendrites and to reduced number of synapses in hippocampal neurons in vitro.

To clarify whether reggie-1 k.o. (Flot2-/-) would affect transport of PSD-95, reggie-1 k.o. (Flot2 - / ) and wt neurons were transfected with PSD-95-EGFP to record vesicle trafficking in living cells. In reggie1 k.o. (Flot2 -/-) neurons, there was a severe defect in the movement of PSD-95 vesicles (Fig. $3 \mathrm{~J}$ ). However, when the overall trafficking of vesicles in these neurons was monitored by phase contrast optics, the activity of the vast majority of moving vesicles appeared normal (Fig. $3 \mathrm{M}, \mathrm{N}$ ). Therefore, the defect seemed to be specific for the carriers containing PSD-95 (and reggie).

\subsubsection{Quantification of vesicle trafficking}

The trafficking of PSD-95-EGFP vesicles was quantified as illustrated in the histogram (Fig. $3 \mathrm{~K}$ ) by monitoring them over $3 \mathrm{~min}$. Whereas PSD-95 vesicle trafficking was significantly reduced in reggie-1 k.o. (Flot2 -/-) versus wt neurons (by 35\%), this reduction was rescued by overexpressing reggie-1-RFP in the k.o. neurons (Fig. $3 \mathrm{~K}$ ). Reggie-1 siRNA-treated wt neurons showed an even stronger reduction (67\%) compared to the control siRNA-treated neurons (Fig. $3 \mathrm{~L}$ ). This reduction in PSD-95 vesicle trafficking in reggie-1 k.o. (Flot2-/-) and siRNA-treated neurons was rescued by reggie-1-RFP transfection and, importantly, was cross rescued by co-transfection with CA-Rab11aRFP (Fig. 3 J, K, L). These results suggest that reggie- 1 and Rab11a are involved in PSD-95 vesicle trafficking, probably involving mediators such as molecular motors (Cornfine et al., 2011).
3.3. Synaptic plasticity and GluA1 (AMPAR1) trafficking in neurons of reggie-1 knock out (Flot2 -/-) mice in vitro

\subsubsection{Reduced GluA1 (AMPAR1) and GluN1 (NMDAR1) in reggie-1 k.o. (Flot2 -/-) neurons}

To determine whether reggie affects an in vitro correlate of synaptic plasticity (in vitro LTP), reggie-1 k.o. hippocampal neurons of postnatal 1-2 d old mice were maturated in vitro to $10 \mathrm{div}$ (and therefore deprived of systemically regulating cues/factors that the brain provides and which influence neuronal maturation in vivo) and tested whether they would exhibit less AMPA GluA1 on the surface and whether this would lead to defects in the glycine-induced correlate of LTP in vitro. The antibody is against an N-terminal extracellular epitope of GluA1 and was previously used in experiments with glycine-induced "in vitro LTP" in mouse hippocampal neurons and proved to preferentially label surface exposed GluA1 under conditions that do not permeabilize cells (4\% PFA, 15 min on ice) (Jurado et al., 2013). Indeed, the in vitro maturated reggie-1 k.o. (Flot2-/-) neurons exhibited 25\% reduced immunostaining intensity for GluA1 in dendrites compared to wt neurons of the same in vitro maturation age (Fig. $4 \mathrm{~A}, \mathrm{~B}$ ).

\subsubsection{Reduced GluA1 and GluN1 in reggie-1 and reggie-2 siRNA treated neurons}

To consolidate this finding, we compared these defects in reggie- 1 k.o. (Flot2 -/-) neurons with effects after acute siRNA-mediated reggie downregulation. Wt hippocampal neurons from 1 to $2 \mathrm{~d}$ old mice were maturated in vitro ( 8 div) and exposed to siRNAs against reggie-1 (alone), or reggie-1 and -2 (combined), or reggie-2 (alone). GluA1 staining in dendrites was reduced by $37 \%$ (reggie- 1 siRNA), by $44 \%$ (reggie-1 and -2 siRNA combined) and by $50 \%$ (reggie-2 siRNA alone) compared to control siRNA-transfected neurons (Fig. 4 C, D) showing that k.o. and siRNA-induced knock down of reggie impairs GluA1 surface exposure in dendrites probably due to the impaired trafficking in reggie deficient neurons.

\subsubsection{Reduced glycine-induced AMPA GluA1 exposure on the cell surface in reggie-1 k.o (Flot2 -/-) and knock down neurons}

When reggie-1 k.o. (Flot2-/-) neurons and wt controls at $10 \mathrm{div}$ were exposed to glycine stimulation to induce LTP in vitro, the GluA1

Fig. 3. Reduced number of synapses, impaired trafficking of PSD-95-EGFP-positive cargo containers and rescue by CA-Rab11a-EGFP in neurons from reggie-1 k.o. (Flot2 -/-) mice.(A) In neurons from 1 to $2 \mathrm{~d}$ old reggie-1 (R1) k.o. (Flot2 - / ) mice process outgrowth was significantly (21\%) delayed compared to $1-2 \mathrm{~d}$ old wt neurons after 2 div but at day 4 , R1 k.o. (Flot2 - / ) neurons were differentiated to the same extent as wt neurons. Scale bar, $20 \mu \mathrm{m}$.(B, C) The histograms show the statistically significant reduction in neurite length at 2 div (average length: $215 \pm 7 \mu \mathrm{m}$ [mean \pm s.e.m.] in wt and $182 \pm 5.8 \mu \mathrm{m}$ [mean \pm s.e.m.] in R1 k.o. neurons, $\mathrm{n}=662$ and $\mathrm{n}=565$, $^{* * *} \mathrm{p}<0.001$ ) $(\mathrm{B})$, and restoration of neurite length at $4 \mathrm{~d}$ [mean \pm s.e.m.] in wt and $720 \pm 24.4 \mu \mathrm{m}$ [mean \pm s.e.m.] in R1 k.o. neurons, $(\mathrm{n}=527$ and $\mathrm{n}=514, \mathrm{p}=0.66 \mathrm{n} . \mathrm{s}$.) (C).(D) Immunostaining with PSD-95 (red) and synaptophysin (Syn, green) Abs (for post and presynaptic elements) on maturated R1 k.o. (Flot2-/-) and wt neurons was reduced in R1 k.o. (Flot2 -/-) neurons over wt controls (D lower row). Upper row shows the PSD-95 immunostaining intensity in black/white. Scale bar, $10 \mu \mathrm{m}$ upper row, $5 \mu \mathrm{m}$ lower row of images.(E, F) Histograms show that PSD-95 staining intensity was $53 \%$ reduced in R1 k.0. (Flot2 - / ) neurons (mean fluorescence intensity $6.72 \pm 1.15$ [mean \pm s.e.m.] in wt and $3.21 \pm 0.67$ [mean \pm s.e.m.] in R1 k.o. neurons, ${ }^{*} \mathrm{p}<0.05$ ) $(\mathrm{E})$. Neurons, $\mathrm{n}=171$ and $\mathrm{n}=179$, in 3 independent experiments. (F) PSD-95 and synaptophysin were 58\% reduced (mean fluorescence intensity: $7.04 \pm 1.6$ [mean \pm s.e.m.] in wt and $3.0 \pm 0.9$ [mean \pm s.e.m.] in R1 k.0. (Flot2 $-/-$ ) neurons, ${ }^{*} \mathrm{p}<0.05$ ). Neurons, $\mathrm{n}=101$ and $\mathrm{n}=104$, in 3 independent experiments. $(\mathrm{G})$ Staining intensity of GluN1 and colocalisation between synaptophysin and GluN1 was reduced in R1 k.o. (Flot2 - / - ) neurons. Upper row shows GluN1 immunostaining intensity in black/white. Scale bar, $10 \mu \mathrm{m}$ upper, $5 \mu \mathrm{m}$ lower row of images.(H, I) Statistically significant reduction in spine synapses in R1 k.o. (Flot2 -/ - ) compared to wt neurons. The staining intensity of GluN1 was $55 \%$ reduced in R1 k.o. (Flot2 $-/-$ ) neurons (14.4 \pm 3.2 [mean \pm s.e.m.] in wt and $6.54 \pm 1.5$ [mean \pm s.e.m.] in R1 k.o neurons, ${ }^{*} \mathrm{p}<0.05$ ). Number of neurons, $\mathrm{n}=114$ and $\mathrm{n}=133$ in 3 independent experiments. The co-localisation of synaptophysin and GluN1 was $42 \%$ reduced $\left(10.7 \pm 1.8\right.$ in wt [mean \pm s.e.m.] and $6.2 \pm 0.8$ [mean \pm s.e.m.] in R1 k.o. (Flot2 $-/-$ ) neurons, $\left.{ }^{*} p<0.05\right)$. Neurons, $\mathrm{n}=111$ and $\mathrm{n}=120$, in 3 independent experiments. $(\mathrm{J})$ Images from $30 \mathrm{~s}$ recordings in a portion of dendrite of R1 k.o. (Flot $2-/-$ ) and wt neurons. The white asterisk to the left marks the same point in all horizontally arranged images. Vesicles (white arrows) move in both directions, away and towards the asterisk. The total distance that the vesicles covered over time is quantified in K. Transport of PSD-95 (white arrows point to individual vesicles) in R1 k.o. (Flot2 - / - ) neurons was 35\% reduced over wt control (histogram (K)). The reduction in trafficking of PSD-95EGFP was partially rescued by CA-Rab11a-RFP and R1-RFP. Scale bar, $2 \mu \mathrm{m}$.(K) Vesicle trafficking was significantly reduced in dendrites of reggie-1 k.o. (Flot2 - / - ) versus dendrites in wt neurons: $8.9 \pm 0.7 \mu \mathrm{m} / \mathrm{min}$ [mean \pm s.e.m.] in wt and $5.9 \pm 0.4 \mu \mathrm{m} / \mathrm{min}$ [mean \pm s.e.m.] in R1 k.o. (Flot $2-/-$ ) neurons, $\mathrm{n}=143$ and $\mathrm{n}=130$, ${ }^{* * *} \mathrm{p}<0.001$. This reduction was rescued by overexpressing R1-RFP in the k.o. (Flot2 - /-) neurons: $8.9 \pm 0.7 \mu \mathrm{m} / \mathrm{min}$ [mean \pm s.e.m.] in wt compared to $8.8 \pm 0.5 \mu \mathrm{m} / \mathrm{min}$ [mean \pm s.e.m.] in R1 k.o. (Flot2 $-/-$ ) neurons, ( $\mathrm{n}=143$ and $\mathrm{n}=139, \mathrm{p}=0.91$, n.s.). CA-Rab11a-RFP also rescued the reduced vesicle transport in dendrites of R1 k.o. (Flot2 $-/-$ ) neurons: $8.9 \pm 0.7 \mu \mathrm{m} / \mathrm{min}[\mathrm{mean} \pm \mathrm{s} . \mathrm{e} . \mathrm{m}$.] in wt and $9.9 \pm 0.8 \mu \mathrm{m} / \mathrm{min}$ (mean \pm s.e.m.) and in R1 k.o. (Flot2 $-/-$ ) dendrites $(\mathrm{n}=143$ and $\mathrm{n}=103, \mathrm{p}=0.7$, n.s.). Vesicles $(\mathrm{n})$ in 3 independent experiments. One-way ANOVA. Multiple Comparisons versus Control Group (Holm-Sidak method).(L) R1 siRNA-treated wt neurons have an even stronger defect in vesicle trafficking in dendrites than R1 k.o. neurons when compared to trafficking in dendrites of control siRNA-treated neurons $(67 \%, 10.4 \pm 0.7 \mu \mathrm{m} / \mathrm{min}$ [mean \pm s.e.m.] in control siRNA and $2.9 \pm 0.1 \mu \mathrm{m} / \mathrm{min}$ in dendrites of reggie-1 siRNA-treated neurons, $\mathrm{n}=147$ and $\mathrm{n}=113{ }^{* * *} \mathrm{p}<0.001$ ). This reduction in PSD-95-EGFP vesicle trafficking in dendrites of R1 siRNA-treated neurons was rescued by R1-RFP transfection $(10.4 \pm 0.7 \mu \mathrm{m} / \mathrm{min}$ [mean \pm s.e.m.] in control siRNA and $8.5 \pm 0.6 \mu \mathrm{m} / \mathrm{min}$ [mean \pm s.e.m.] in R1siRNA-treated neurons, $\mathrm{n}=147$ and $\mathrm{n}=118, \mathrm{p}=0.08$, n.s.), and, importantly, was cross-rescued by co-transfection with CA-Rab11a-RFP $(10.4 \pm 0.7 \mu \mathrm{m} / \mathrm{min}$ [mean \pm s.e.m.] in control siRNA and $9.4 \pm 0.6 \mu \mathrm{m} / \mathrm{min}$ [mean \pm s.e.m.] in R1 siRNA treated neurons, $\mathrm{n}=147$ and $\mathrm{n}=150, \mathrm{p}=0.25$, n.s.). Vesicles $(\mathrm{n})$ in 3 independent experiments. One-way ANOVA. Multiple Comparisons versus Control Group (Holm-Sidak method).(M) Trafficking vesicles (three examples are indicated by black, red, and green arrows) in wt and R1 k.o. (Flot2-/-) neurites in phase contrast move in both directions (see positions of the arrows).(N) The average vesicle trafficking rate was similar in wt and R1 k.o. (Flot $2-/-)$ neurons $(44.76 \pm 8.3 \mu \mathrm{m} / \mathrm{min}$ [mean $\pm \mathrm{s} . \mathrm{e} . \mathrm{m}$.] in wt and $46.4 \pm 3.4 \mu \mathrm{m} / \mathrm{min}$ [mean \pm s.e.m.] in R1 k.o. neurons (Flot2 $-/-$ ), $\mathrm{n}=107$ and $\mathrm{n}=101, \mathrm{p}=0.86$, n.s.). Vesicles $(\mathrm{n})$ in 2 independent experiments. 
A

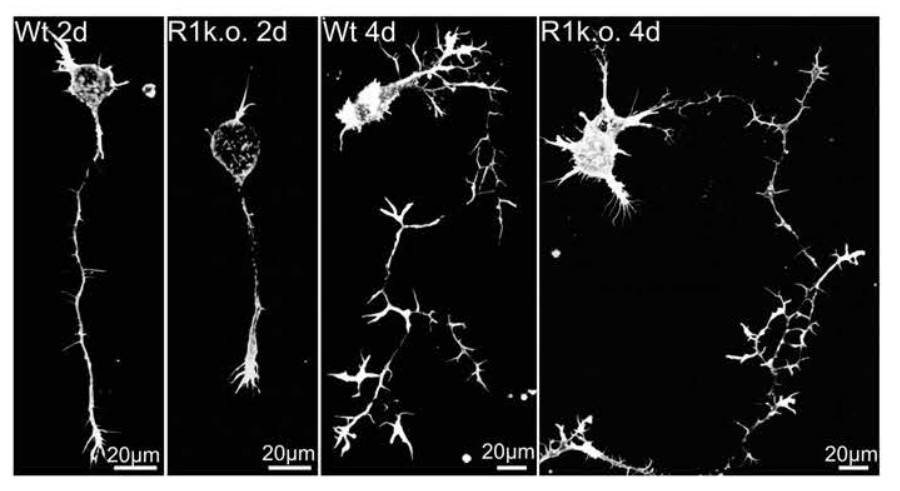

D

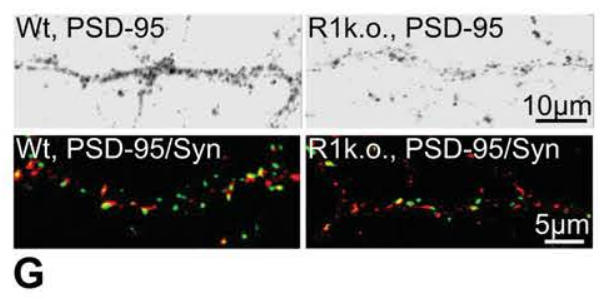

Wt, GluN11 R1k.o., GluN1

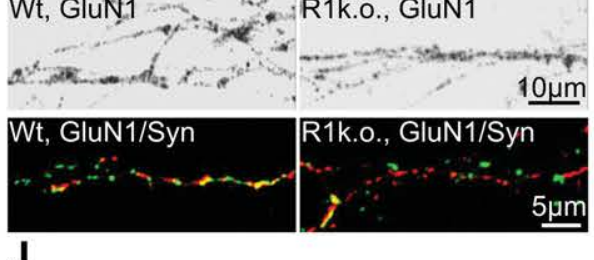

E
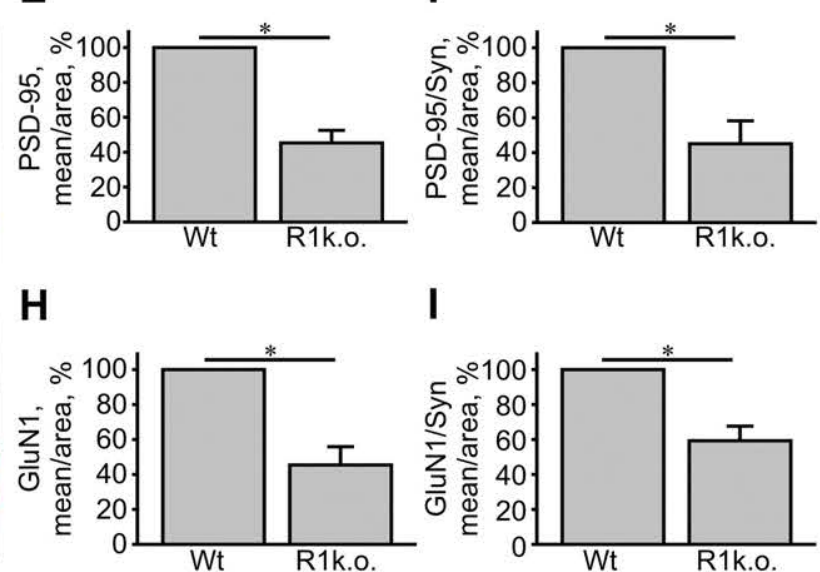

B

Wt 2d R1k.o. 2d

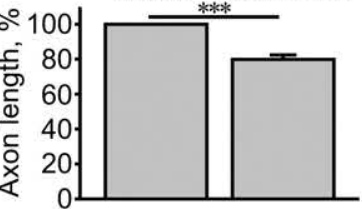

C

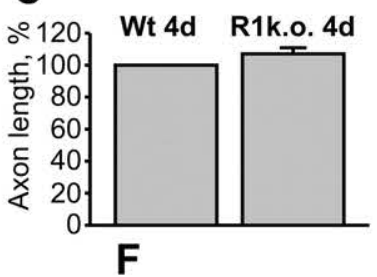

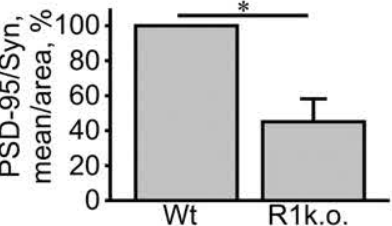

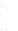

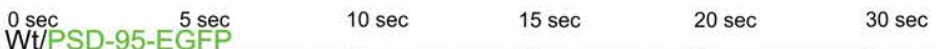

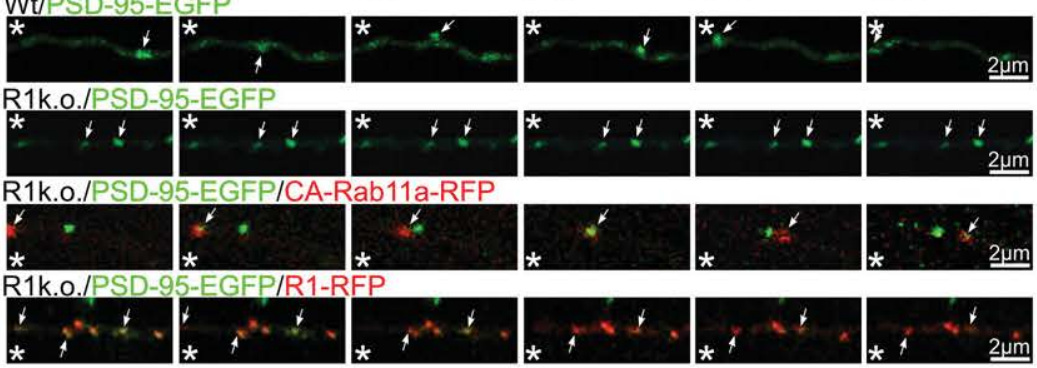

K

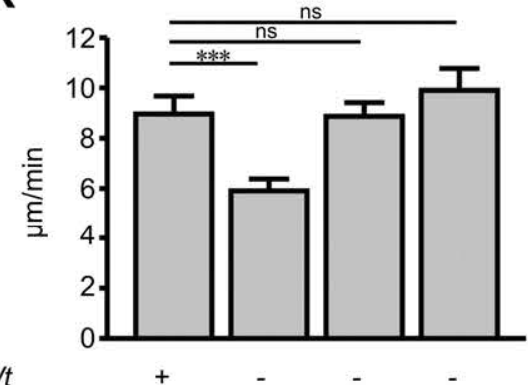

L

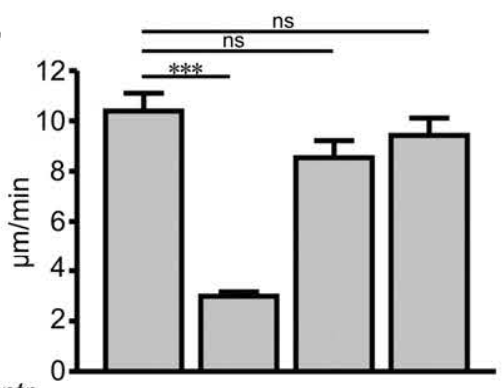

Wt

PSD-95

$-E G F P$

R1-RFP

CA-Rab11a

Contr

SiRNA
R1

SiRNA

PSD-95

-EGFP

R1-RFP

CA-Rab
-RFP 

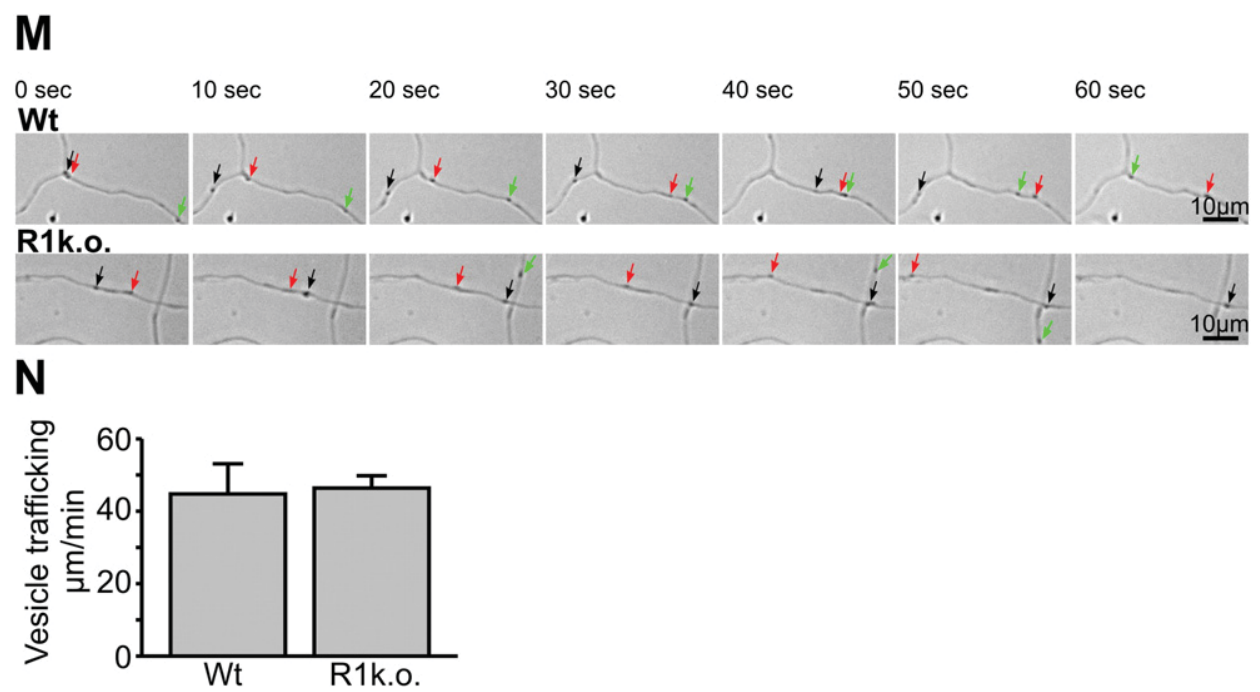

Fig. 3 (continued).

staining increased in wt neurons by $66 \%$, but not in the k.o. counterparts (Fig. 4 A, B). Dendrites in reggie-1 k.o. (Flot2-/-) neurons had 61\% less GluA1 on the surface than wt controls after exposure to glycine (Fig. 4 A, B) probably because of trafficking defects after loss of reggie-1 (see Fig. 6). When reggie-1(alone), reggie-1 and reggie-2 (combined) or reggie2 (alone) siRNA-treated neurons were stimulated with glycine to induce LTP, the GluA1 staining intensity fell by $26 \%$, by $43 \%$ and by $40 \%$, respectively, over control siRNA-transfected and unstimulated neurons. But this difference was even larger with glycine-stimulated control siRNA transfected neurons which showed a $45 \%$ increase in GluA1 staining intensity over age-matched unstimulated control siRNA transfected neurons (Fig. 4 C, D). Thus, reggie-1 and reggie-2 promote GluA1 delivery to spines which is considered as an in vitro correlate of LTP (Jurado et al., 2013) and synaptic plasticity in vitro.

When reggie-1 and reggie-2 siRNA-treated neurons were cotransfected with CA-Rab11a-EGFP and then exposed to glycine stimulation, the intensity of GluA1 increased dramatically (Fig. 4 C, D) consistent with the notion that the recycling machinery is important in the induction of synaptic plasticity (Gerges et al., 2004, Park et al., 2006) and the in vitro correlate of LTP.

These data would imply that reggie-1 k.o. mice might exhibit abnormalities in behavior unless regulatory mechanisms become activated to compensate such defects. As adult reggie- 1 k.o. (and reggie- 1 and -2 double k.o. (Flot1 -/-; Flot2-/-)) mice have no overt phenotype (Berger et al., 2013; Bitsikas et al., 2014a,b) and normal in vivo LTP (http://cms.uni-konstanz.de/stuermer/research/supplementary-data$\mathrm{ltp} /$ ) we investigated whether signs of such compensation can be found in the brain.

3.4. Regulation of signaling and adhesion proteins as well as AMPA GluA1 and NMDA GluN1 in the brains of young and adult reggie-1 k.o. (Flot2 -/-) mice

To determine whether there are measurable defects in young $1-2 \mathrm{~d}$ old reggie-1 k.o. (Flot $2-/-$ ) brains (at stages at which the neurons are isolated and raised in vitro) and whether correlative expressional changes occur during differentiation in vivo, we performed quantitative Western blots of $2 \mathrm{~d}$ old wt and reggie-1 k.o. (Flot2 -/-) brains (Fig. 5 A, C, E, G, I, K, M, O, Q), and compared them to 2 months old wt and reggie-1 k.o. (Flot2 -/-) brains (Fig. 5 B, D, F, H, J,L, N, P, R). In 1-2 d old reggie-1 k.o. (Flot2-/-) brains there was no change in MAPK and pMAPK (Fig. 5 A) but a 63\% increase in psrc (Fig. 5 C), a 62\% decrease of pPSD-95 and 26\% decrease of PSD-95 (Fig. 5 E), a 25\% increase in JNK1 but $40 \%$ decrease in pJNK1 (Fig. $5 \mathrm{I}$ ), and 19\% decrease in $\mathrm{N}$ cadherin (Fig. 5 G). GluA1 and GluN1 were hardly detectable in either, young wt and k.o. brains. Thus, at young age, reggie-1 k.o. (Flot2-/-) brains exhibit severe defects in the regulation of signal transduction molecules (psrc, pJNK1) and the cell adhesion protein N-cadherin which are important for neurite elongation and synaptogenesis (Arikkath and Reichardt, 2008). Rab11a was unchanged in $2 \mathrm{~d}$ (Fig. 5 O) and 2 months old brains (Fig. 5 P). Young reggie-1 k.o. (Flot2-/-) brains also display reduced levels of PSD-95 which is involved in dendrite differentiation (Charych et al., 2006) and the development of spines.

In 2 months reggie- 1 k.o. (Flot2-/-) brains, by contrast, pMAPK was 55\% decreased compared to wt (Fig. 5 B) whereas significant increase was seen for $\operatorname{src}(60 \%$, Fig. 5 D), PSD-95 (35\%, Fig. 5 F), N-cadherin (72\%, Fig. 5 H) and GluA1 (40\%, Fig. 5 L). GluN1 was decreased (54\%, Fig. 5 $\mathrm{N})$. The misregulation of the above proteins in reggie-1 k.o. (Flot2-/-) brains seems to correspond to the structural and functional deficits in neurons from young reggie-1 k.o. (Flot2 -/-) mice in vitro, i.e., delay in process extension, impaired transport of PSD-95, and reduced number of synapses.

Thus, the upregulation of N-cadherin, PSD-95 and GluA1 and downregulation of GluN1 in 2 months reggie-1 k.o. (Flot2 - /-) mice suggests that mechanisms in the brain counterregulate in reggie-1 k.o. (Flot2-/-) mice the expressional changes of proteins involved in LTP that occurred in the young brains. In addition, the reduced expression levels of pMAPK and increased src in adult reggie k.o. brains is indicative of severe and longlasting influences on many targets since pMAPK and psrc are involved in many signaling cascades including those involved in synapse formation (Thomas and Huganir, 2004). Thus, significant regulation of specific proteins is occurring in the reggie-1 k.o. (Flot2-/-) brain, which could explain why mice do not have an overt phenotype, and why adult reggie-1 k.o. (Flot2 -/-) mice have normal LTP.

These data suggest that reggies are important co-regulators of glycine-induced and GluN1-mediated transport and targeting of GluA1 in spines (which is an indicator of in vitro LTP). These results support the view that reggie-1 and reggie-2 together with Rab11a promote the recycling of vesicular carriers with cargo destined for the PSD: reggie and Rab11a are important for PSD-95 trafficking (Fig. 6). It seems that the same Rab11a and reggie-coated vesicular carriers contain in addition to PSD-95, N-cadherin, GluA1 and GluN1 which speaks for dendritic transport of packages whose targeted delivery to the PSD depends on reggie and Rab11a.

\section{Discussion}

Our present results show a function of reggies in spine synapse formation and demonstrate their influence on an in vitro correlate of LTP in 
hippocampal neurons in vitro. This function was detected in reggie-1 k.o. (Flot2-/-) as well as reggie siRNA-treated neurons where we found impaired trafficking of PSD-95, and reduced N-cadherin, GluA1 and GluN1 delivery to spines. The trafficking defect, reduced number of spine synapses and impairment of an in vitro correlate of LTP in

A

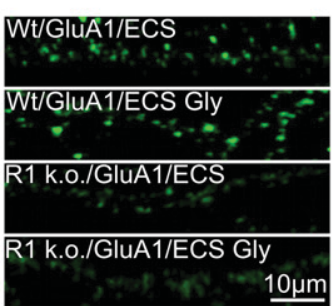

C
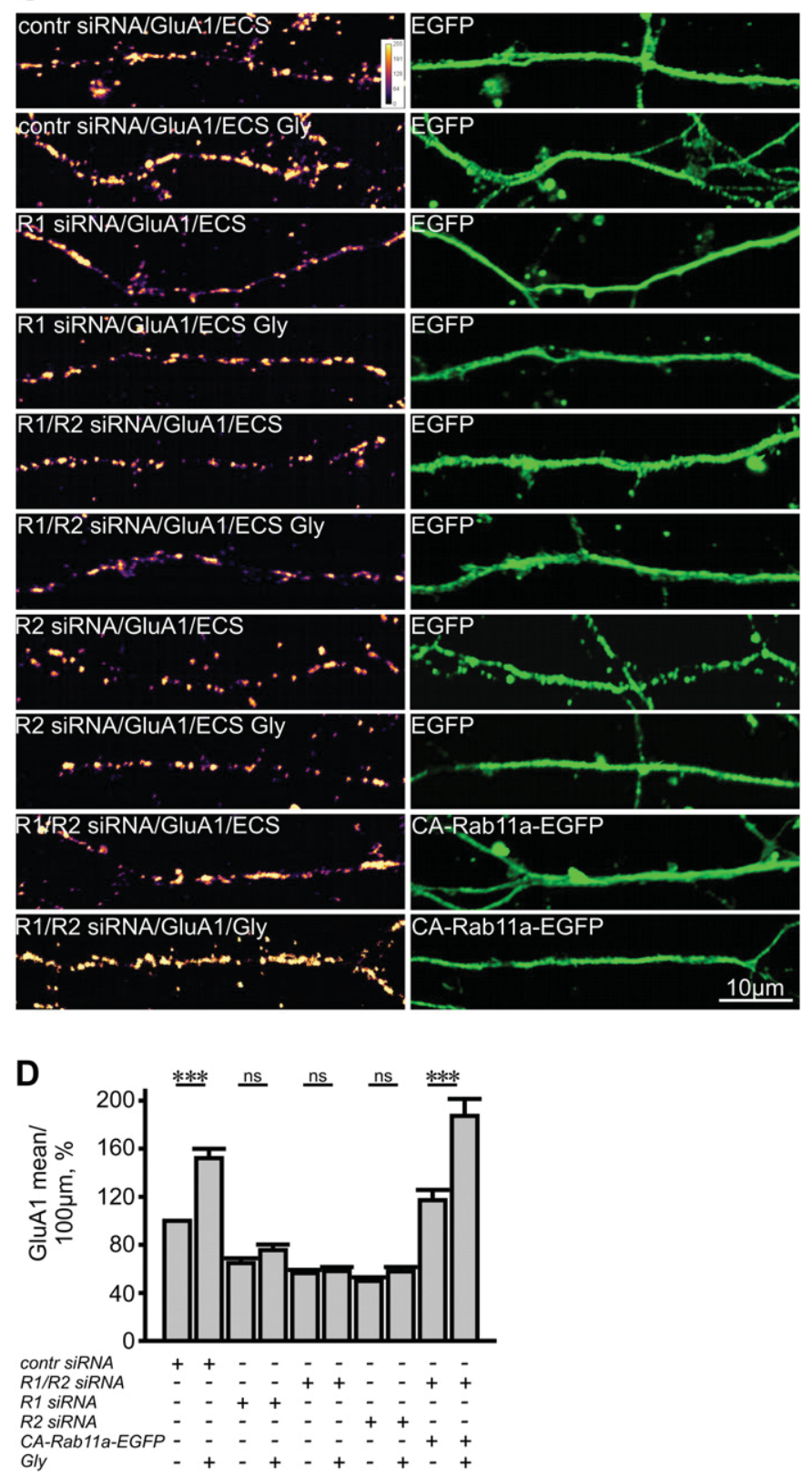

reggie-deprived and reggie-1 k.o. (Flot2-/-) neurons were partially rescued by CA-Rab11a and reggie-1 supporting that Rab11a and reggies are functional partners in the same trafficking pathway (Solis et al., 2013), promoting the targeted delivery of cargo to spines.

How the obvious defects of reggie- 1 k.o. (Flot2 $-/-$ ) neurons in vitro relate to the phenotypically normal reggie-1 k.o. (Flot2-/-) mouse and normal LTP in vivo was assessed by the comparative analysis of adult k.o. and wt brains in Western blots. This revealed significant alterations in signaling pathways, upregulation of N-cadherin, PSD-95, GluA1 (and their phosphorylated forms) in k.o. brains and downregulation of GluN1 - all known to be involved in spine synapse formation and LTP (Kennedy and Ehlers, 2011). The detected changes (together with unidentified ones) might counteract in a complex manner the defects in cargo trafficking and recycling in reggie-1 k.o. (Flot2-/-) mice, implying that the reggie-1 k.o. mutant might be "cured" by compensation. Already young ( $1-2 \mathrm{~d}$ ) reggie- 1 k.o. (Flot $2-/-$ ) brains had significantly reduced levels of the proteins involved in spine synapse formation such as PSD-95 (and its phosphorylated form) and N-cadherin, and showed levels and activation of signal transduction molecules (src, JNK) that markedly differed from 1 to $2 \mathrm{~d}$ wt brains. These changes in reggie- 1 k.o. (Flot $2-/-$ ) brains imply early onset of compensatory mechanisms and longlasting adaptations. Regulation of genes and protein modification as well as changes in the activation of signaling molecules in reggie-1 k.o. animals complies with recent reports showing that knock out of specific genes leads to seemingly normal mice through compensation of expressional and activity regulating factors (Barbaric et al., 2007; Rossi et al., 2015, below). Importantly, reggies regulate together

Fig. 4. Impaired in vitro LTP in reggie-1 k.o. (Flot2-/-) hippocampal neurons. (A) Hippocampal neurons from wt and reggie-1 (R1) k.o. (Flot2-/-) mice were maturated in vitro (10 d), exposed to glycine in ECS (ECS Gly) or ECS only, and subjected to live immunolabeling with $\mathrm{Ab}$ against the extracellular domain of GluR1. The fluorescence intensity of GluR1 was determined over dendrites, portions of which are shown in A and C. Scale bar, $10 \mu \mathrm{m}$. (B) The quantification of immunofluorescence intensity with and without the addition of glycine ( Gly + and Gly -, respectively) and statistical significance values are indicated in the histogram. AMPA GluR1 staining is $25 \%$ reduced in R1 k.o. neurons (Flot $2-/-$ ) compared to wt controls $(48.1 \pm 5.3$ [mean + s.e.m.] in wt and $36.2 \pm 3.04$ [mean \pm s.e.m.] in reggie-1 k.o. (Flot2-/-) neurons, $\mathrm{n}=67$ and $\mathrm{n}=73,{ }^{*} \mathrm{p}<0.01$ ). There is a significant increase in AMPA GluR1 staining after stimulation with ECS glycine over ECS alone in wt neurons (by 66\%, $48.1 \pm 5.3$ [mean \pm s.e.m.] in wt and $80.1 \pm 10.3$ [mean \pm s.e.m.] in wt with Gly,$+ \mathrm{n}=67$ and $\mathrm{n}=80,{ }^{*} \mathrm{p}<0.01$ ) but not in R1 k.o. (Flot2 $-/-$ ) neurons ( $36.2 \pm 3.04$ [mean \pm s.e.m.] in reggie-1 k.o. (Flot2 $-/-$ ) neurons and $31.7 \pm 3.5$ [mean \pm s.e.m.] in reggie-1 k.o. (Flot2-/-) with Gly,$+ \mathrm{n}=73$ and $\mathrm{n}=78, \mathrm{p}=0.62$, n.s.). Importantly, there is a reduction of $61 \%$ when wt neurons treated with Gly + are compared to R1 k.o. (Flot2 - / -$)$ neurons treated with Gly $+(80.11 \pm 10.3$ [mean \pm s.e.m.] in wt/ Gly + and $31.7+3.5$ [mean + s.e.m.] in R1 k.o. (Flot $2-/-$ ) / Gly + neurons, $n=80$ and $\mathrm{n}=78,{ }^{* * *} \mathrm{p}<0.001$ ). Neurons ( $\left.\mathrm{n}\right)$ from 3 independent experiments. One-way ANOVA. Pairwise Comparisons between Groups (Holm-Sidak method). (C) Hippocampal neurons from wt mice were maturated in vitro $(10 \mathrm{~d})$ and treated with control siRNA (Contr siRNA) or reggie-1 siRNA (R1siRNA) or reggie-1 and reggie-2 siRNAs (R1/R2 siRNA) combined (left column). Neurons were cotransfected with EGFP (right column) or CA-Rab11a-EGFP in the last two images and exposed to glycine in ECS (ECS Gly) or ECS only. Scale bars, $10 \mu \mathrm{m}$. To highlight intensity changes, images were processed by Image (LUT, mpl-inferno), with blue, representing low intensity, and yellow/white, high intensity. (D) The histogram shows the quantification of surface GluA1 under the different experimental conditions and statistical significance. Glycine increased surface GluA1 staining in control siRNA-treated neurons (intensity: $24.1 \pm$ 1.15 [mean \pm s.e.m.] in control siRNA and $36.5 \pm 1.85$ [mean \pm s.e.m.] in control siRNA/Gly treated neurons, $\mathrm{n}=129$ and $\mathrm{n}=136,{ }^{*} * * \mathrm{p}<0.001$ ) but not in neurons treated with reggie-1 (R1) siRNA (15.5 \pm 0.9 [mean \pm s.e.m.] in R1 siRNA and $18.1 \pm$ 1.1 [mean \pm s.e.m.] in R1siRNA/Gly treated neurons, $\mathrm{n}=132$ and $\mathrm{n}=143, \mathrm{p}=0.89$, n.s.), and, respectively, with reggie-2 (R2) siRNA $(12.0 \pm 0.74$ [mean \pm s.e.m.] in R2 siRNA and $13.9 \pm 0.87$ [mean \pm s.e.m.] in R2 siRNA/Gly treated neurons, $\mathrm{n}=117$ and $\mathrm{n}=99, \mathrm{p}=0.97$, n.s.) or with R1/R2 siRNA combined (13.5 \pm 0.65 [mean \pm s.e.m.] in $\mathrm{R} 1 / \mathrm{R} 2$ siRNA and $14.0 \pm 0.77$ [mean \pm s.e.m.] in R1/R2 siRNA/Gly treated neurons, $\mathrm{n}=$ 134 and $\mathrm{n}=136, \mathrm{p}=0.99$, n.s.). In CA-Rab11a-EGFP-treated neurons glycine significantly increased surface GluA1, compared to CA-Rab11a-EGFP-treated unstimulated neurons by $60 \%(28.1 \pm 2.6$ [mean \pm s.e.m.] in R1/2 siRNA and $44.9 \pm 3.3$ [mean \pm s.e.m.] in R1/2 siRNA/Gly treated neurons, $\mathrm{n}=102$ and $\mathrm{n}=116$, $\left.{ }^{* * *} \mathrm{p}<0.001\right)$. Neurons $(\mathrm{n})$ in 3 independent experiments. One-way ANOVA. Pairwise Comparisons between Groups (Holm-Sidak method). 
with Rab11a recycling and transport of cargo destined for spine synapse formation (and plasticity) and cause, when missing, dramatic counteractive expressional changes in brain.

We were able to identify abnormalities resulting from reggie- 1 k.o. (Flot2 - / ) and downregulation by live imaging in cultured neurons: neurite extension was delayed in young k.o. neurons and PSD-95 trafficking was severely impaired in neurons maturated to 10 div in vitro. This observation suggested that PSD-95 is transported in reggie/ Rab11a carriers. Since vesicles with reggie-1, Rab11a and PSD-95 also contain N-cadherin, GluA1 and GluN1, our observations suggest that transport of multiprotein packages destined for the PSD (Park et al.,
2006) is impaired in the absence of reggie- 1 resulting in reduced number of synapses and reduced dendritic and spine localisation of GluN1 and GluA1 which is a correlate of abnormal in vitro LTP. It is puzzling that we observe an impairment of the correlate of LTP in neurons in vitro but not in vivo, possibly because compensatory factors support neuronal development within the brain which are absent in vitro.

It has been suggested that reggies participate in endocytosis of raftassociated proteins (Glebov et al., 2006) but this view was not supported in many other subsequent experiments including our own (Langhorst et al., 2005; Stuermer, 2010; Solis et al., 2013; Hülsbusch et al., 2015) and was recently revised by the same lab which has

$2 d$

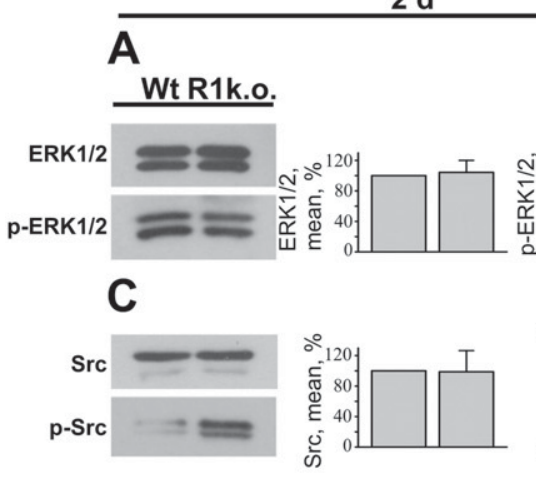

$\mathbf{E}$
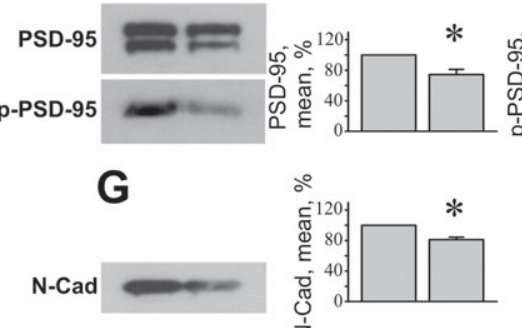

I
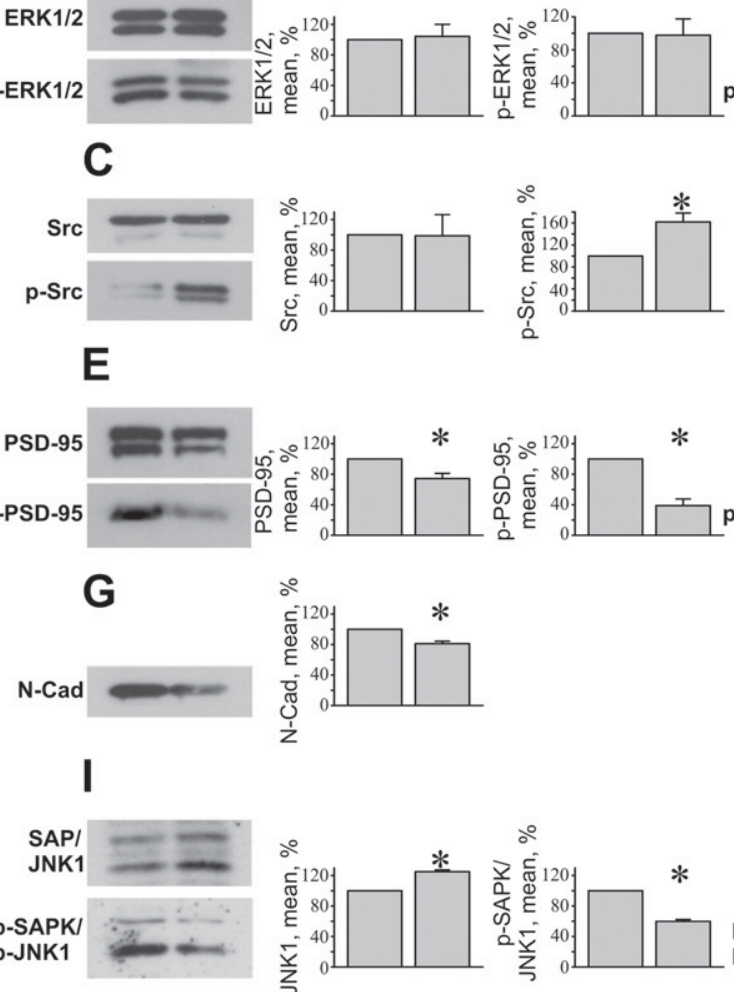
p-ERK1/2
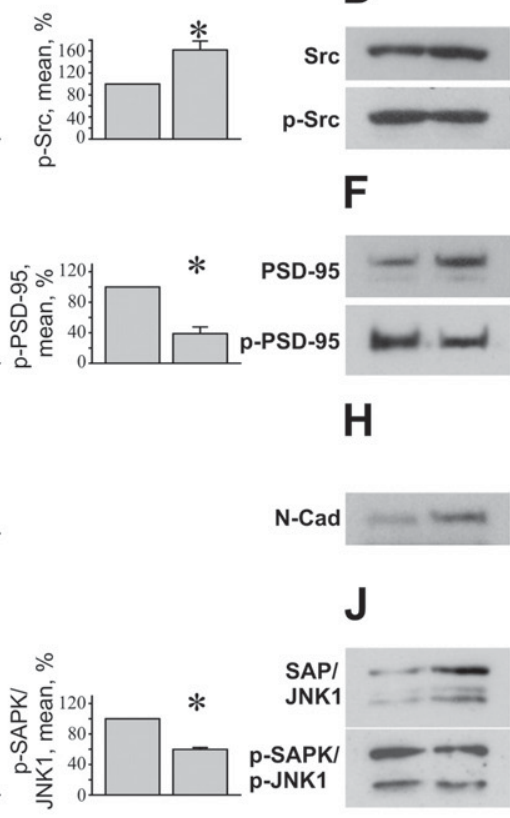

a

D

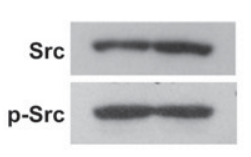

$\mathbf{F}$

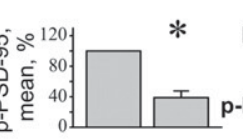

p-PSD-95
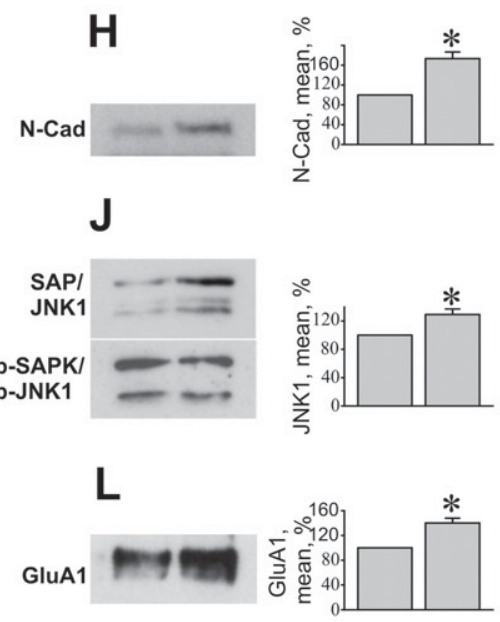

2 months

B

\section{Wt R1k.o.}

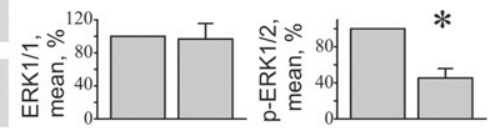

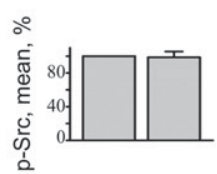
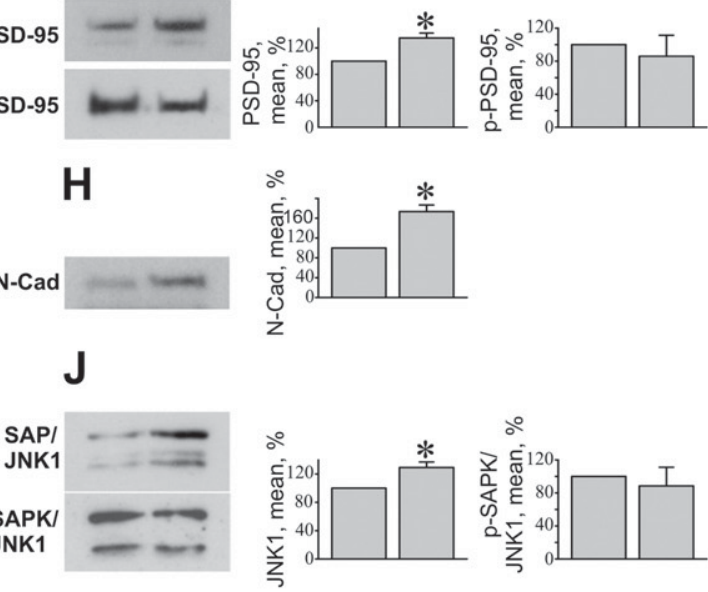

K

GluA1
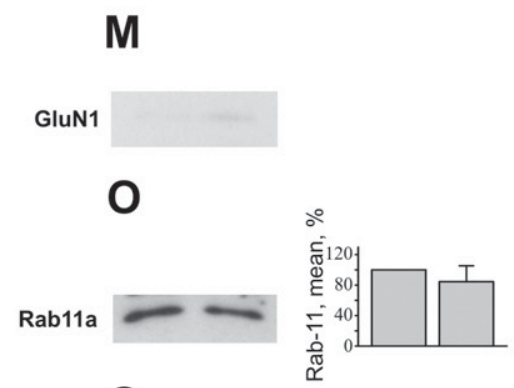

Q

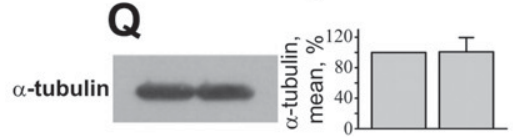

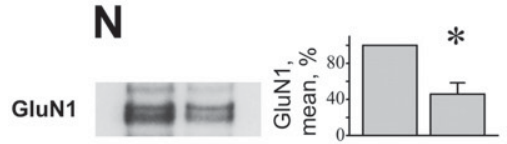

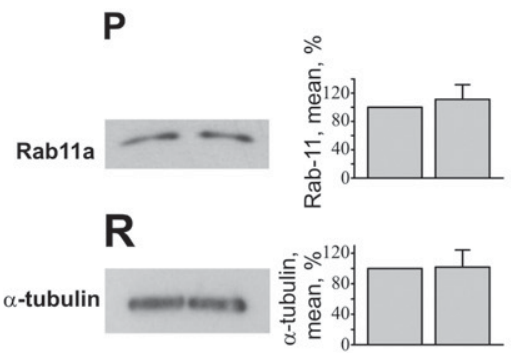


published it initially (Bitsikas et al., 2014a,b). Thus, it is unlikely that reggie downregulation impairs endocytosis of the FM4-64X dye which serves as a measure of synapses in our study. Other presynaptic effects of reggie downregulation are possible (in connection with recycling) and would require further analysis. However, PSD-95 which acts postsynaptically can overcome the loss of synapses after reggie downregulation which speaks for the fact that reggie downregulation indeed perturbs the cargo transport to the postsynaptic spine.

Our work implies that reggies are important constituents of cargo containers destined for the PSD in hippocampal neurons. Reggies function as coregulators of cargo trafficking and delivery in neurons (Bodrikov et al., 2011) much as in non-neuronal cells where reggies were involved in Rab11a-dependent trafficking and the site-specific delivery of E-cadherin to adherens junctions (Solis et al., 2013) and $\alpha 5$ and $\beta 1$-integrins to focal adhesions (Hülsbusch et al., 2015).

\subsection{Correlative expressional changes in reggie-1 k.o. (Flot2 -/-) brains}

The phenomenon that mice have no overt phenotype after knock out of specific genes is quite common (Rossi et al., 2015; Barbaric et al., 2007) and is explained by compensation. A recent publication showed that genetic loss of molecular regulators of GluA1 trafficking and LTP can be compensated by cGMP-dependent protein kinase II (cGKII) which preserves phosphorylation of GluA1 by blocking calcineurin. cGKII k.o. mice showed no impairment of LTP whereas LTP was impaired when cGKII was acutely inhibited (Kim et al., 2015). The LTP in k.o. mice was mimicked as it involved $\mathrm{Ca}^{2+}$ permeable GluA1. Importantly, this work by Kim and colleagues shows network compensation after genetic deletion of cGKII. The complex changes in protein expression levels and signal transduction molecules in reggie-1 k.o. (Flo2-/) versus wt brains correlate with the development towards normal brain functions and suggests that compensatory network regulation might become activated in the present reggie-1 k.o. (Flot2-/-) mice as well and change dynamically from postnatal to 2 months old brains, as evidenced by the comparative quantitative immunoblot analysis.

A possible compensatory protein for the loss of reggie-1 in the reggie-1 k.o. (Flot2 -/-) mouse could have been reggie-2 but reggie2 rather occurred at lower levels in the reggie-1 k.o. brain. Compensation by Rab11a upregulation was also not seen. Reggies also interact with TC10 (Kioka et al., 2002; Bodrikov et al., 2011) and influence expression and activation of other Rho family GTPases such as Rac1 (Hülsbusch et al., 2015) which might compensate the defects.

Interestingly, the reggie- 1 and -2 (flotillin-2 and -1) double k.o. mouse has no significant phenotype either (Bitsikas et al., 2014a,b) nor has the loss-of-function mutation of reggie-1 and reggie-2 in Drosophila led to significant defects in the fly's brain (Hoehne et al., 2005). This was surprising since reggies/flotillins are present in basically every cell type and are evolutionarily extremely well conserved (from bacteria to man) which predicts that they subserve conserved and important functions. However, reggie overexpression in wing imaginal disks severely disrupted wing development (Hoehne et al., 2005) by affecting secretion and spreading of wnt and hedgehog. Reggie-1 overexpression in wing disks perturbed the gradient of the morphogens (Katanaev et al., 2008). In rats upregulation of reggie-1 in adult retina ganglion cells (RGCs) promoted axon regeneration (Koch et al., 2013), and interestingly, downregulation of injury-induced reggie upregulation in fish RGCs reduced axon regeneration (Munderloh et al., 2009). These results together with impaired growth of reggie-1 k.o. hippocampal neurons in vitro and an impaired in vitro correlate of synaptic plasticity (in vitro LTP) are consistent with reggies playing a role in cargo trafficking and delivery which is needed for growth and plasticity.

It was reported that reggie-2 overexpression in maturated hippocampal neurons leads to increased spines and EPSPs (Swanwick et al., 2010). Another study showed by mass spectrometry that reggies were also upregulated during memory related strengthening of synapses in Aplysia (Monje et al., 2013) and during barrel cortex plasticity (Butko et al., 2013). These studies are consistent with the present results and emphasize the role of reggie in brain function and synaptic plasticity. The findings by Swanwick et al. (2010) and our own results suggest that reggie-2 may have an even stronger influence on spine formation than reggie-1. Reggie-2 downregulation reduced spine number by $59 \%$, and reggie- 1 siRNA by $24 \%$. It would, therefore, be interesting to investigate the role of reggie- 2 in neurons and brains of reggie-2 k.o. (Flot $1-/-$ ) mice in future studies.

\subsection{Reggie and Rab11a-dependent cargo trafficking}

The trafficking vesicles which are affected by reggie-1 k.o. (Flot2-/-) and knock down belong to the recycling compartment. Recycling endosomes are known to serve as AMPA receptor (GluA) reservoir in spines (Newpher and Ehlers, 2008). This complies with our results from Hela and A431 cells where reggie- 1 and reggie-2 reside at the Rab11a-positive tubulo-vesicular recycling compartment and directly interact with Rab11a (Solis et al., 2013). Accordingly, CA-Rab11a in the former and present study partially rescued the reggie- 1 k.o. and knock down defects, here, by restoring the number of spines and spine synapses.

It has been described in axons that specific cargo for synapses is transported as pre-fabricated packages (Ahmari et al., 2000) along microtubules into the axon terminals. A similar trafficking mechanism involving myosin Va and/or b, binding to Rab11a, delivers PSD-associated cargo to dendritic spines (Correia et al., 2008; Wang et al., 2008). Park et al. (2004) and Gerges et al. (2004) have demonstrated that spine growth and PSD formation depend on the entry of Rab11a dependent

Fig. 5. Regulation of signaling molecules and PSD-associated proteins in brains from $2 \mathrm{~d}$ old wt and reggie-1 k.o. (Flot2 - /-) mice, compared to brains from 2 months old wt and reggie- 1 k.o. mice. Signals on Western blots for the indicated proteins were quantified over three independent experiments and depicted in the histograms to the right with indications of statistically significant differences between the pairs (2-tailed unpaired Student's $t$-test). Left column: $2 \mathrm{~d}$ wt and reggie-1 (R1) k.o. (Flot2 - / ) brains, right column: 2 months wt and k.o. (Flot2-/-) brains. (A) 2 d old reggie-1 k.o. (Flot2-/-) brains showed no change in ERK1/2 (19.3 \pm 2.5 [mean \pm s.e.m.] in wt and $20.2 \pm 3.06$ [mean \pm s.e.m.] in reggie-1 k.o. brains, $\mathrm{p}=0.84$, n.s.) or pERK1/2 (16.9 \pm 3.5 in wt [mean \pm s.e.m.] and $16.5 \pm 3.3$ [mean \pm s.e.m.] in reggie-1 k.o. brains, $\mathrm{p}=0.93$, n.s.). (B) There was a statistical significant $55 \%$ decrease in pERK1/2 in 2 months reggie-1 k.o. (Flot2 -/-) brains ( $7.7 \pm 1.1$ [mean \pm s.e.m.] in wt and $3.5 \pm 0.8$ [mean \pm s.e.m.] in reggie-1 k.o. brains, ${ }^{*} \mathrm{p}<0.05$ ). (C) There was a $63 \%$ statistical significant increase in p-src in 2 d reggie-1 k.o. (Flot2 -/-) brains ( $11.7 \pm 1.2$ [mean \pm s.e.m.] in wt and $18.9 \pm 1.9$ [mean \pm s.e.m.] in reggie- 1 k.o. brains, $\left.{ }^{*} \mathrm{p}<0.05\right)$. (D) Src (but not p-src) was increased (statistically significant) by $60 \%$ in reggie-1 k.o. (Flot2 $-/-$ ) brains at 2 months $(9.4 \pm 1.2$ [mean \pm s.e.m.] in wt and $15.07 \pm 1.04$ [mean \pm s.e.m.] in reggie-1 k.o. brains, ${ }^{*}$ p < 0.05). (E) 2 d reggie-1 k.o. (Flot2 -/-) brains had a 26\% decrease (statistically significant) of PSD-95 (9.5 \pm 0.68 [mean \pm s.e.m.] in wt and $7.1 \pm$ 0.66 [mean \pm s.e.m.] in reggie-1 k.o. brains, ${ }^{*}$ p $\left.<0.05\right)$ and $62 \%$ decrease (statistically significant) of pPSD-95 $(22.03 \pm 4.4$ [mean \pm s.e.m.] in wt and $8.5 \pm 1.9$ [mean \pm s.e.m.] in reggie-1 k.o. brains, ${ }^{*} \mathrm{p}<0.05 \%$ ). (F) PSD-95 (but not pPSD-95) was increased (statistically significant) in 2 months reggie-1 k.o. (Flot2 -/ - ) brains (35\%, $11.9 \pm 1.4$ [mean \pm s.e.m.] in wt and $16.1 \pm 0.9$ [mean \pm s.e.m.] in reggie-1 k.o. brains, ${ }^{*} \mathrm{p}<0.05$ ). (G) There was a $19 \%$ decrease (statistically significant) of N-cadherin in $2 \mathrm{~d}$ old reggie- 1 k.o. (Flot2 $-/-$ ) brains $\left(8.8 \pm 0.5\right.$ [mean \pm s.e.m.] in wt and $7.2 \pm 0.2$ [mean \pm s.e.m.] in reggie-1 k.o. brains, $\left.{ }^{*} \mathrm{p}<0.05\right)$. (H) N-cadherin was $73 \%$ increased (statistically significant) in 2 months reggie-1 k.o. (Flot $2-/-$ ) brains $\left(4.4 \pm 0.8\right.$ [mean \pm s.e.m.] in wt and $7.6 \pm 0.5$ [mean \pm s.e.m.] in reggie- 1 k.o. brains, ${ }^{*}$ p $\left.<0.05\right)$. (I) SAPK/JNK1 was $25 \%$ (statistically significant) increased ( $10.9 \pm 0.7$ [mean \pm s.e.m.] in wt and $13.6 \pm 0.2$ [mean \pm s.e.m.] in reggie-1 k.o. brains, ${ }^{*} \mathrm{p}<0.05$ ) but pSAPK1/JNK1 was $41 \%$ decreased (statistically significant) in $2 \mathrm{~d}$ reggie-1 k.o. (Flot2 -/-) brains (19.1 \pm 2.5 [mean \pm s.e.m.] in wt and $11.4 \pm 0.4$ [mean \pm s.e.m.] in reggie-1 k.o. brains, ${ }^{*}$ p $<0.05$ ). (J) SAPK/JNK1 (but not its phosphorylated form) was increased (statistically significant) in 2 months reggie-1 k.o. (Flot $2-/-)$ brains $(29 \%, 6.52 \pm 0.4$ [mean \pm s.e.m.] and $8.4 \pm 0.5$ [mean \pm s.e.m.] in wt and reggie-1 k.o. brains, respectively, ${ }^{*} \mathrm{p}<0.05$ ). (K) GluA1 was hardly detectable in $2 \mathrm{~d}$ brains. (L) GluA1 was significantly upregulated in reggie-1 k.o. (Flot $\left.2-/-\right)$ compared to wt brains ( $40 \%$, $4.2 \pm 0.6$ [mean \pm s.e.m.] in wt and $5.9 \pm 0.3$ [mean \pm s.e.m.] in reggie-1 k.o. brains, $\left.{ }^{*} \mathrm{p}<0.05\right)$. (M) GluN1 was hardly detectable in young wt and reggie-1 k.o. (Flot2 $-/-$ ) brains. (N) GluN1 is present in 2 months brains but at significantly reduced levels in reggie- 1 k.o (Flot $2-/-)$ compared to wt brains ( $54 \%, 11.3 \pm 2$ [mean \pm s.e.m.] in wt and $5.2 \pm 1.4$ [mean \pm s.e.m.] in reggie-1 k.o. brains, $\left.{ }^{*} \mathrm{p}<0.05\right)$. (O, P) Rab11a was not changed. (Q, R) $\alpha$-Tubulin served as loading controls. Quantitative values (A-P) were normalized to $\alpha$-tubulin. 


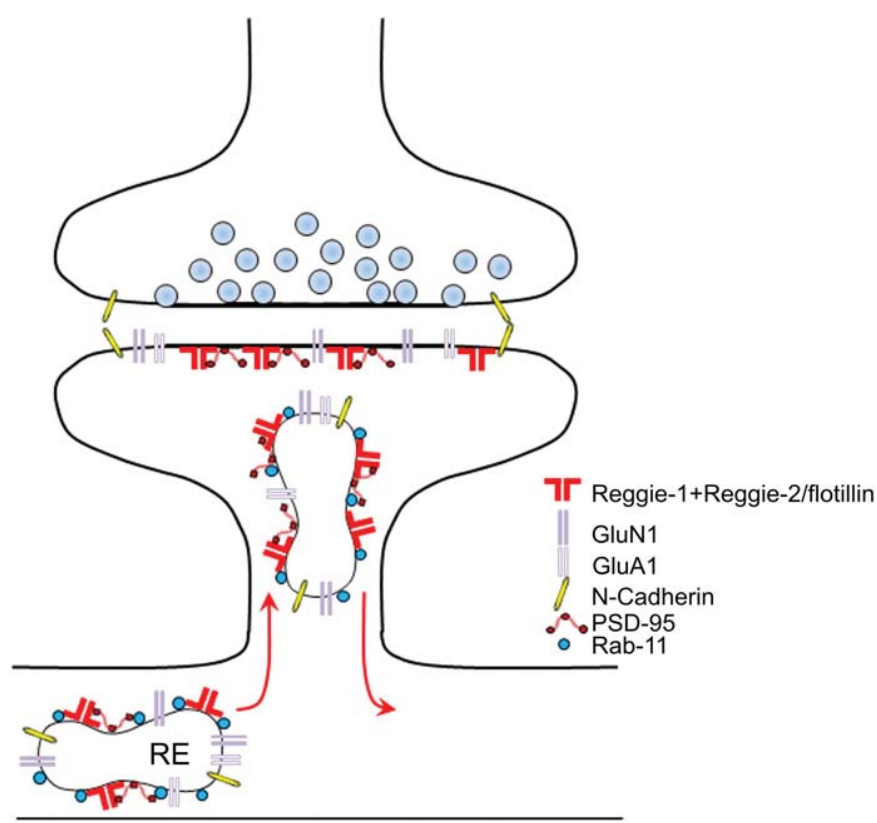

Fig. 6. Schematic representation of reggie-coated Rab11a positive transport containers in dendrites and spines for the delivery of cargo to the PSD. The reggie-coated Rab11a positive cargo vesicles (RE) co-transport PSD-95, N-cadherin, the glutamate receptors GluA1 and GluN1 for delivery to the postsynaptic membrane in spines of hippocampa neurons (red arrows).

recycling endosome into spines in which AMPA GluAs are delivered to the PSD. The reggie-coated vesicles with PSD-95, N-cadherin, AMPA GluA1 and NMDA GluN1 seem to belong to the spine-directed recycling endosome. The immunoglobulin superfamily protein Telencephalin (which is proposed to stabilize spines) is also transported in reggie-2/ flotillin-1 labeled containers (Raemaekers et al., 2012).

\subsection{Reggies in rafts}

It is possible that the components of the cargo vesicles favor the same specific lipid environment as reggies and that the modification fatty acid acylations found in reggies, PSD-95 (El-Husseini et al., 2000) and GluA1 and the lipid raft association of N-cadherin (Suzuki et al., 2011) facilitates their assembly or transport in reggie-coated carriers. In this model, reggies appear to create or demarcate membrane domains of specific lipid composition, probably like EHD1 and SNX4 which are residents of the same recycling compartment in Hela and A431 cells as the reggies and which are associated with phosphatidylinositol-4-phosphate and phosphatidylinositol-4,5bisphosphate (PIP2) (Jovic et al., 2009; Cullen, 2011; Solis et al., 2013). Different phospho-inositides are implied in (post)synaptic plasticity and AMPA receptor (GluA) recycling (Leitner et al., 2015). Conceivably then, reggies might organize microdomains with specific lipids and lipid modified proteins and promote their vesicular transport and delivery to specific regions such as the PSD. This complies with a recent report showing that dynein clusters in reggie/flotillin microdomains during transport and maturation of phagosomes to lysosomes in Leishmania donovani (Rai et al., 2016).

\section{Conclusion}

The reggies are important constituent of the recycling endosome which is known to be required for spine synapse formation and function (Park et al., 2004, 2006; Gerges et al., 2004) by promoting the targeted delivery of cargo.

\section{Acknowledgement}

This work was supported by a grant from the Deutsche Forschungsgemeinschaft to C.A.O.S. (Stu-112/32-1)

\section{References}

Ahmad, M., Polepalli, J.S., Goswami, D., Yang, X., Kaeser-Woo, Y.J., Südhof, T.C., Malenka, R.C., 2012. Postsynaptic complexin controls AMPA receptor exocytosis during LTP. Neuron 73, 260-267.

Ahmari, S.E., Buchanan, J., Smith, S.J., 2000. Assembly of presynaptic active zones from cytoplasmic transport packets. Nat. Neurosci. 3, 445-451.

Arikkath, J., Reichardt, L.F., 2008. Cadherins and catenins at synapses: roles in synaptogenesis and synaptic plasticity. Trends Neurosci. 31, 487-494.

Barbaric, I., Miller, G., Dear, T.N., 2007. Appearances can be deceiving: phenotypes of knockout mice. Brief. Funct. Genomic. Proteomic. 6, 91-103.

Berger, T., Ueda, T., Arpaia, E., Chio, I.I., Shirdel, E.A., Jurisica, I., Hamada, K., You-Ten, A. Haight, J., Wakeham, A., Cheung, C.C., Mak, T.W., 2013. Flotillin-2 deficiency leads to reduced lung metastases in a mouse breast cancer model. Oncogene 32, 4989-4994.

Bitsikas, V., Corrêa, I.R., Nichols, B.J., 2014a. Clathrin-independent pathways do not contribute significantly to endocytic flux. eLife 3, e03970 (2014).

Bitsikas, V., Riento, K., Howe, J.D., Barry, N.P., Nichols, B., 2014b. The role of flotillins in regulating $A ß$ production, investigated using flotillin $1-/-$, flotillin $2-/-$ double knockout mice. PLoS One 9, e85217.

Bodrikov, V., Solis, G.P., Stuermer, C.A., 2011. Prion protein promotes growth cone development through reggie/flotillin-dependent N-cadherin trafficking. J. Neurosci. 31 18013-18025.

Brown, T.C., Correia, S. S, Petrok, CN. Esteban, JA 2007. Functional compartmentalization of endosomal trafficking for the synaptic delivery of AMPA receptors during longterm potentiation. J. Neurosci. 28, 13311-13315.

Butko, M.T., Savas, J.N., Friedman, B., Delahunty, C., Ebner, F., Yates, J.R., Tsien, R.Y., 2013. In vivo quantitative proteomics of somatosensory cortical synapses shows which protein levels are modulated by sensory deprivation. Proc. Natl. Acad. Sci. USA 110, E726-E735.

Charych, E.I., Akun, B.F., Goldberg, J.S., Jörnsten, R.J., Rongo, C., Zheng, J.Q., Firestein, B.L., 2006. Activity-independent regulation of dendrite patterning by postsynaptic density protein PSD-95. J. Neurosci. 26, 10164-19176.

Cornfine, S., Himmel, M., Kopp, P., El-Azzouzi, K., Wiesner, C., Krüger, M., Rudel, T. Linder, S., 2011. The kinesin KIF9 and reggie/flotillin proteins regulate matrix degradation by macrophage podosomes. Mol. Biol. Cell 22, 202-215.

Correia, S.S., Bassani, S., Brown, T.C., Lise, M.-F., Bockos, D.S., El-Husseini, A., Passafaro, M. Esteban, J.A., 2008. Motor protein-dependent transport of AMPA receptors into spines during long-term potentiation. Nat. Neurosci. 11, 457-466.

Cullen, P.J., 2011. Phosphoinositides and the regulation of tubular-based endosomal sorting. Biochem. Soc. Trans. 39, 839-850.

El-Husseini, A.E., Craven, S.E., Chetkovich, D.M., Firestein, B.L., Schnell, E., Aoki, C., Bredt D.S., 2000. Dual palmitoylation of PSD-95 mediates its vesiculotubular sorting, postsynaptic targeting and ion channel clustering. J. Cell Biol. 148, 159-172.

Gerges, N.Z., Backos, D.S., Esteban, J.A., 2004. Local control of AMPA receptor trafficking at the postsynaptic terminal by a small GTPase of the Rab family. J. Biol. Chem. 279, 43870-43878.

Glebov, O.O., Bright, N.A., Nichols, B.J., 2006. Flotillin-1 defines a clathrin-independent endocytic pathway in mammalian cells. Nat Cell Biol 8, 46-54.

Hoehne, M., de Couet, H.G., Stuermer, C.A.O., Fischbach, K.F., 2005. Loss- and gain-of-function analysis of the lipid raft proteins Reggie/Flotillin in Drosophila: they are posttranslationally regulated, and misexpression interferes with wing and eye development. Mol. Cell Neurosci. 30, 326-338.

Hülsbusch, N. Solis, G.P., Katanaev, V.L., Stuermer, C.A., 2015. Reggie-1/Flotillin-2 regulates integrin trafficking and focal adhesion turnover via Rab11a. Eur. J. Cell Biol. 94, 531-545.

Jovic, M., Kieken, F., Naslavsky, N., Sorgen, P.L., Caplan, S., 2009. Eps15 homology domain 1-associated tubules contain phosphatidylinositol-4-phosphate and phosphatidylinositol-(4,5)-bisphosphate and are required for efficient recycling. Mol. Biol. Cell 20, 2731-2743.

Jurado, S., Goswami, D., Zhang, Y., Molina, A.J., Südhof, T.C., Malenka, R.C., 2013. LTP requires a unique postsynaptic SNARE fusion machinery. Neuron 77, 542-558.

Katanaev, V.L., Solis, G.P., Huasmann, G., Buestorf, S., Katanayeva, N., Schrock, Y., Stuermer C.A.O., Basler, K., 2008. Reggie-1/flotillin-2 promotes secretion of the long-range signaling forms of Wingless and Hedgehog in Drosophila. EMBO J. 27, 509-521.

Kennedy, M.J., Ehlers, M.D., 2011. Mechanisms and function of dendritic exocytosis. Neuron $69,856-875$.

Kim, S., Titcombe, R.F., Zhang, H., Khatri, L., Girma, H.K., Hofmann, F., Arancio, O., Ziff, E.B., 2015. Network compensation of cyclic GMP-dependent protein kinase II knockout in the hippocampus by $\mathrm{Ca}^{2+}$-permeable AMPA receptors. PNAS 112, 3122-3127.

Kioka, N., Ueda, K., Amachi, T., 2002. Vinexin, CAP/ponsin, ArgBP2: a novel adaptor protein family regulating cytoskeletal organization and signal transduction. Cell Struct. Funct. $27,1-7$.

Koch, J., Solis, G.P., Bodrikov, V., Michel, U., Haralampieva, D., Shypitsyna, A., Tönges, L., Bähr, M., Lingor, P., Stuermer, C.A.O., 2013. Upregulation of reggie-1/flotillin-2 promotes axon regeneration in the rat optic nerve in vivo and neurite growth in vitro. Neurobiol. Dis. 51, 168-176.

Langhorst, M., Reuter, A., Stuermer, C.A.O., 2005. Scaffolding microdomains and beyond the function of reggie/flotillin proteins. Mol. Cell. Life Sci. 62, 2228-2240.

Leitner, M.G., Halaszovich, C.R., Ivanova, O., Oliver, D., 2015. Phosphoinositide dynamics in the postsynaptic membrane compartment: mechanisms and experimental approach. Eur. J. Cell Biol. 94, 401-414. 
Monje, F.J., Divisch, I., Demit, M., Lubec, G., Pollak, D.D., 2013. Flotillin-1 is an evolutionaryconserved memory-related protein up-regulated in implicit and explicit learning paradigms. Ann. Med. 45, 301-307.

Munderloh, C., Solis, G.P., Bodrikov, V., Jaeger, F.A., Wiechers, M., Málaga-Trillo, E., Stuermer, C.A., 2009. Reggies/flotillins regulate retinal axon regeneration in the zebrafish optic nerve and differentiation of hippocampal and N2a neurons. J. Neurosci. 29, 6607-6615.

Newpher, T.M., Ehlers, M.D., 2008. Glutamate receptor dynamics in dendritic microdomains. Neuron 58, 472-497.

Park, M., Penick, E.C., Edwards, J.G., Kauer, J.A., Ehlers, M.D., 2004. Recycling endosomes supply AMPA receptors for LTP. Science 305, 1972-1975.

Park, M., Salgado, J.M., Ostroff, L., Helton, T.D., Robinson, C.G., Harris, K.M., Ehlers, M.D., 2006. Plasticity-induced growth of dendritic spines by exocytic trafficking from recycling endosomes. Neuron 52, 817-830.

Raemaekers, T., Peric, A., Baatsen, P., Sannerud, R., Declerck, I., Baert, V., Michiels, C., Annaert, W., 2012. ARF6-mediated endosomal transport of Telencephalin affects dendritic filopodia-to-spine maturation. EMBO J. 31, 3252-3269.

Rai, A., Pathak, D., Thakur, S., Singh, A., Dubey, A.K., Mallik, R., 2016. Dynein clusters into microdomains on phagosomes to drive rapid transport toward lysosomes. Cell 164, 722-734.

Rossi, A., Kontarakis, Z., Gerri, C., Nolte, H., Hölper, S., Krüger, M., Stainier, D.Y., 2015. Genetic compensation induced by deleterious mutations but not gene knockdowns. Nature 524, 230-233.

Schulte, C., Racchetti, G., D'Alessandro, R., Meldolesi, J., 2010. A new form of neurite outgrowth sustained by the exocytosis of enlargeosomes expressed under the control of REST. Traffic 10, 1304-1314.

Solis, G.P., Hoegg, M., Munderloh, C., Schrock, Y., Málaga-Trillo, E., Rivera-Milla, E., Stuermer, C.A.O., 2007. Reggie/flotillin proteins are organized into stable tetramers in membrane microdomains. Biochem. J. 403, 313-322.
Solis, G.P., Málaga-Trillo, E., Plattner, H., Stuermer, C.A., 2010. Cellular roles of the prion protein in association with reggie/flotillin microdomains. Front. Biosci. 15 1075-1085.

Solis, G.P., Hülsbusch, N., Radon, Y., Katanaev, V.L., Plattner, H., Stuermer, C.A., 2013. Reggies/flotillins interact with Rab11a and SNX4 at the tubulovesicular recycling compartment and function in transferrin receptor and E-cadherin trafficking. Mol. Biol. Cell 24, 2689-2702.

Stuermer, C.A., 2010. The reggie/flotillin connection to growth. Trends Cell Biol. 20, 6-13. Stuermer, C.A., Langhorst, M.F., Wiechers, M.F., Legler, D.F, Von Hanwehr, S.H. Guse, A.H. Plattner, H., 2004. PrPc capping in T cells promotes its association with the lipid raft proteins reggie-1 and reggie-2 and leads to signal transduction. FASEB J. 18, $1731-1733$.

Suzuki, T., Zhang, J., Miyazawa, S., Liu, Q., Farzan, M.R., Yao, W.-D., 2011. Association of membrane rafts and postsynaptic density: proteomics, biochemical, and ultrastructural analyses. J. Neurochem. 119, 64-77.

Swanwick, C.C., Shapiro, M.E., Vicini, S., Wenthold, R.J., 2010. Flotillin-1 promotes formation of glutamatergic synapses in hippocampal neurons. Dev. Neurobiol. 13, 875-883.

Takahashi, S., Kubo, K., Waguri, S., Yabashi, A., Shin, H.W., Katoh, Y., Nakayama, K., 2012 Rab11 regulates exocytosis of recycling vesicles at the plasma membrane. J. Cell Sci. $125,4049-4057$.

Thomas, G.M., Huganir, R.L., 2004. MAPK cascade signalling and synaptic plasticity. Nat. Rev. Neurosci. 5, 173-183.

Wang, Z., Edwards, J.G., Riley, N., Provance, D.W., Karcher, R., Li, X.D., Davison, I.G., Ikebe, M., Mercer, J.A., Kauer, J.A., Ehlers, M.D., 2008. Myosin Vb mobilizes recycling endosomes and AMPA receptors for postsynaptic plasticity. Cell 135, 535-548. 